\title{
Minimal redefinition of the OSV ensemble
}

\author{
Shahrokh Parvizi, Alireza Tavanfar ${ }^{\dagger}$ \\ Institute for Studies in Theoretical Physics and Mathematics (IPM), \\ P.O.Box 19395-5531, Tehran, Iran
}

November 13, 2018

\begin{abstract}
In the interesting conjecture, $\mathrm{Z}_{\mathrm{BH}}=\left|\mathrm{Z}_{\mathrm{top}}\right|^{2}$, proposed by Ooguri, Strominger and Vafa (OSV), the black hole ensemble is a mixed ensemble and the resulting degeneracy of states, as obtained from the ensemble inverse-Laplace integration, suffers from prefactors which do not respect the electric-magnetic duality. One idea to overcome this deficiency, as claimed recently, is imposing nontrivial measures for the ensemble sum.

We address this problem and upon a redefinition of the $O S V$ ensemble whose variables are as numerous as the electric potentials, show that for restoring the symmetry no non-Euclidean measure is needful. In detail, we rewrite the OSV free energy as a function of new variables which are combinations of the electric-potentials and the black hole charges. Subsequently the Legendre transformation which bridges between the entropy and the black hole free energy in terms of these variables, points to a generalized ensemble. In this context, we will consider all the cases of relevance: small and large black holes, with or without $D_{6}$-brane charge. For the case of vanishing $D_{6}$-brane charge, the new ensemble is pure canonical and the electric-magnetic duality is restored exactly, leading to proper results for the black hole degeneracy of states. For more general cases, the construction still works well as far as the violation of the duality by the corresponding OSV result is restricted to a prefactor. In a concrete example we shall show that for black holes with nonvanishing $D_{6}$-brane charge, there are cases where the duality violation goes beyond this restriction, thus imposing non-trivial measures is incapable of restoring the duality. This observation signals for a deeper modification in the OSV proposal.
\end{abstract}

IPM/P-2005/059

*Email: parvizi@theory.ipm.ac.ir

†Email: art@ipm.ir 


\section{Introduction}

The theory of topological strings is a beautiful, smart and powerful mathematics to probe various aspects of the superstring theories. From the time of its invention [1][2][3], it has been an active field of new discoveries. The reader may refer to [4] as an excellent textbook on the topological strings, or consult [5][6][7] as quick introductions to the subject and [5] for a nice view to the recent developments.

On the other hand, one of the most striking outcomes of the string theory has been the microscopic derivation of the black hole entropy [8]. Recently Ooguri, Strominger and Vafa (OSV) in [9] have proposed a deep and promising connection between the topological free energy and the microstate ensemble of the 4-dimensional BPS Black Holes in the $C Y_{3}$ compactifications of type-II strings. This conjecture roots from earlier works on the black hole entropy [10][11][12] which made a consistent generalization of the BekensteinHawking formula, incorporating the F-terms corrections to the supergravity action as encoded in the prepotential. The evidence for the fact that a generalization to the standard area-law formula for the black hole entropy is needful in the context of string-M theory had been provided in [13]. A detailed review on the results about the black hole entropy from the string theory is [14].

In [9], OSV observe that the Bekenstein-Hawking-Wald entropy of the black holes, as derived in [11][12], can be re-expressed as the Legendre transformation of a real function $\mathcal{F}$, called the OSV free energy, with respect to the half of its variables,

$$
\mathrm{S}\left(\mathrm{p}^{\Lambda}, \mathrm{q}_{\Lambda}\right)=\mathcal{F}\left(\mathrm{p}^{\Lambda}, \phi^{\Lambda}\right)+\mathrm{q}_{\Lambda} \phi^{\Lambda} \quad ; \quad \mathcal{F}\left(\mathrm{p}^{\Lambda}, \phi^{\Lambda}\right) \equiv-\pi \Im \mathrm{F}\left(\mathrm{CX}^{\Lambda}, \mathrm{C}^{2} \mathrm{~W}^{2}=2^{8}\right)
$$

with

$$
\mathrm{CX}^{\Lambda}=\mathrm{p}^{\Lambda}+\mathrm{i} \phi^{\Lambda}
$$

as obtained from the magnetic part of the attractor equations, and $\mathrm{F}\left(\mathrm{X}^{\Lambda}\right)=\mathrm{F}\left(\mathrm{X}^{0}, \mathrm{X}^{\mathrm{A}}\right)$ being the full holomorphic prepotential. Independently, a very close relation is established between the asymptotic expansion of the prepotential and the free energy of topological strings [15][16]. In all the OSV conjecture states that, given a BPS black hole of electricmagnetic charge-multiplet $\left(p^{\Lambda}, q_{\Lambda}\right)$, arising in $\mathcal{N}=2$ compactification of $I I A(B)$ string theory on $C Y_{3}=\mathcal{M}$, the topological $A-\bar{A}(B-\bar{B})$ models on $\mathcal{M}$ define a mixed ensemble as,

$$
\mathrm{Z}_{\text {mix }} \equiv \mathrm{e}^{\mathcal{F}\left(\mathrm{p}^{\Lambda}, \phi^{\Lambda}\right)}=\sum_{\mathrm{q}_{\Lambda}} \Omega\left(\mathrm{p}^{\Lambda}, \mathrm{q}_{\Lambda}\right) \mathrm{e}^{-\pi \mathrm{q}_{\Lambda} \phi^{\Lambda}}
$$

where the black hole free energy is given by

$$
\mathrm{F}_{\mathrm{BH}}=\mathcal{F}\left(\mathrm{p}^{\Lambda}, \phi^{\Lambda}\right)=\mathrm{F}_{\text {top }}+\overline{\mathrm{F}}_{\text {top }}
$$

with $\Omega\left(\mathrm{p}^{\Lambda}, \mathrm{q}_{\Lambda}\right)$ proposed as (index) degeneracy of the black hole states and $\phi^{\Lambda}$ being the electric potential corresponding to $\mathrm{q}_{\Lambda} \cdot{ }^{*}$ This proposal has been successfully tested in [18]

${ }^{*}$ For a non-perturbative completion of the original conjecture see [17], also there are suggestions concerning the holomorphic anomaly [18][19][20]. 
and [21] from different points of view and further developed in [17]. Remarkably using the OSV conjecture [21] was able for the first time to derive from the macroscopic side, the exact coefficient of the leading term in the asymptotic expansion of the degeneracy of states for the small black hole of type-IIA on $K_{3} \times T^{2}$, dual to Heterotic on $T^{6}$, where both the prepotential and the microscopic-degeneracy counting are known exactly. For a review lecture on [9] [18] and [17], see [22].

There are however examples in [23][24] where a naive application of this conjecture sounds problematic. For example [23] mainly focuses on $\mathcal{N}=4$ models where in some cases the string coupling is strong. Before concentrating on the concern of this paper, it is worth to point out that before any try to apply or to refine the proposal one may note that the OSV conjecture in form of [9][17] is a statement about the full free energy of the topological string theory, including all the perturbative quantum corrections as well as the non-perturbative contributions. Therefore regarding the fact that the non-perturbative completion of the topological free energy is not known so far, what the OSV conjecture provides is so a non-perturbative definition of the topological strings, parallel to the program of topological M-theory as proposed in [25] and [26].

A distinguished ambiguity in (1.3) originates from the electric-magnetic duality considerations, that is the requirement of the invariance of $\Omega\left(p^{I}, q_{I}\right)$ under the (relevant subgroup) of the symplectic transformations. Actually as concrete examples show [23][27][24], given the relevant terms of the topological free energy, the inverse Laplace transformation corresponding to $(1.3)^{\dagger}$,

$$
\Omega\left(\mathrm{p}^{\Lambda}, \mathrm{q}^{\Lambda}\right) \doteq \int\left[\mathrm{d} \phi^{\Lambda}\right] \mathrm{e}^{\mathcal{F}+\pi \mathrm{q}_{\Lambda} \phi^{\Lambda}} \equiv \int\left[\mathrm{d} \phi^{\Lambda}\right] \mathrm{e}^{\mathrm{G}}
$$

does lead to the results which are duality violating due to some unwanted prefactors, for a highlighted example of which see the next section . As we will argue later, this property is not restricted to a specific subset of prepotentials but it is a general fact about (1.5). Modifying this deficiency has been a matter of some investigations. For example, as a try within an independent and, by construction, duality invariant formulation see [28], where the proposed modification works for the dyonic black holes relatively well but it is not applicable to the case of $\frac{1}{2} \mathrm{BPS}$ states for which the proposed measure vanishes. Alternatively [29][30][31] propose a black hole ensemble which sums over a single dualityinvariant charge, so by construction respects the expected symmetries. The application of this approach, where the non-holomorphic corrections to the free energy should necessarily be considered, is so far limited to $\frac{1}{2} \mathrm{BPS}$ black holes. So there is not yet any overlap between these two approaches. Another idea to improve the duality violations, being closer to the OSV proposal, is assuming that the OSV free energy intrinsically lives in a curved space, accordingly (1.5) is redefined via a non-Euclidean measure [24][20],

$$
\bar{\Omega}\left(\mathrm{p}^{\Lambda}, \mathrm{q}_{\Lambda}\right) \doteq \int\left[\mathrm{d} \phi^{\Lambda}\right] \sqrt{|\mathrm{g}(\mathrm{p}, \phi)|} \mathrm{e}^{\mathcal{F}+\pi \mathrm{q}_{\Lambda} \phi^{\Lambda}}
$$

This measure which differs from case to case is responsible for removing the mentioned prefactors. Along this path, [24] calls for a "deeper understanding of the integration

\footnotetext{
$\dagger$ The sign $\doteq$ means " equality " up to an arbitrary constant which is independent of the black hole charges.
} 
measure implicit in (1.5)".

In this paper we focus on this problem, that is the duality aspects of the OSV conjecture. We shall prove, at least asymptotically, the converse of the statement which leads to (1.6). That is, the curvature of the space where the OSV free energy is defined, asymptotically or exactly, is zero. To show this we shall follow a direct approach: explicit construction of a proper flat ensemble, based on the OSV free energy, which is canonic in as many variables as the original OSV ensemble. In different examples, also within general arguments, we observe that the ensemble leads to the proper results for the black hole degeneracy of states. In fact the construction works properly for all types of the known BPS black holes in $C Y_{3}$ compactifications of type-IIA(B) theories and to all orders in the saddle point asymptotic expansion of the inverse Laplace integral, as far as the duality violation is restricted to a prefactor. This is done via a simple change of variables which being linear in the electric-potentials, preserves the Legendre transformation from the free energy to the entropy. The new variables have the advantage that the corresponding ensemble is potentially protected against the duality violating prefactors. For the cases with vanishing $D_{6}$-brane charge, where the prepotential takes a simplified form and the construction is readable from the form of the prepotential, the new ensemble is pure canonical and restores the electric-magnetic exactly. The idea still works quite well for the most general case, of course up to nonholomorphic corrections which are missed also in the original OSV proposal. For the most general case, considering the leading term of the saddle-point approximation, we obtain a general constraint on the Jacobian from the new variables to the old ones. This constraint-equation admits infinite number of solutions, in principle all the solutions are equally well, but practically we can pick up the simplest choices. In fact the exact choice of these variables is not needed. The job they do is just removing the duality-violating prefactor of the corresponding OSV result, which could be done by hand from the first. In this sense it is an existence problem.

It is worth to ask about higher orders of the saddle-point approximation for the most general case. The question is whether the duality violation appears just as a prefactor in higher orders. Indeed, for the black holes with vanishing $\mathrm{D}_{6}$-brane charge the answer is positive to all orders of the asymptotic expansion, however we will show that in case of non-vanishing $\mathrm{D}_{6}$-brane charge, the violation can become more complicated than a simple prefactor. This implies that, in higher orders, a simple measure factor is not sufficient to restore the duality generally and deeper modifications/investigations are necessary.

Finally as an alternative, motivated by manifest symplectic-invariant construction, we try a different ensemble which from the beginning treats both of the magnetic and electric charges at the same footing. This enlarged ensemble is related to the ensemble of section 4 via an effective integration over the magnetic potentials and asymptotically reproduces the same results for the black hole degeneracy of states.

The outline of the paper is as follows. In the next section, for the clearness of the main problem within a concrete example, we review one result from [23][24]. In section 3, we redefine the OSV ensemble for the large and small black holes in the absence of $D_{6}$-brane, respectively, and show how it leads to proper results for the degeneracy of states. In section 4 we lift the idea for the most general case of BPS black holes in the context of $C Y_{3}$ compactifications of type-IIA(B) string theories. In section 5, we check out the procedure for higher orders in the saddle-point approximation and show that in some 
special cases the duality violation can not be fixed by a single prefactor. In section 6 we introduce an effective which asymptotically reproduces the same results of section 4 . We end the paper with a conclusion.

\section{Electric-magnetic duality in the OSV conjecture}

Let us start by addressing the duality invariance of the proposal of [9]. To do that, we repeat swiftly a result from [21], [23] and $[24]^{\ddagger}$. The prepotential of type $I I$ string theory on a $C Y_{3}$ is given by the free energy of the corresponding topological string theory as follows :

$$
\mathrm{F}_{\mathrm{II}}=-\frac{\mathrm{i} \pi}{2} \mathrm{~F}_{\text {top }} \quad \mathrm{F}_{\text {top }}=\mathrm{F}_{\text {top }}^{\text {pert. }}+\mathrm{F}_{\text {top }}^{\mathrm{GW}}+\mathrm{F}_{\text {top }}^{\text {non-pert. completion }}
$$

where

$$
\mathrm{F}^{\text {pert. }}=\sum_{\mathrm{h}} \mathrm{F}_{\mathrm{h}} \mathrm{W}^{2 \mathrm{~h}}
$$

with $F_{h}$ being the genus- $h$ amplitude of the topological theory and $W^{2}$ includes the graviphoton field strength. For the case of IIA on $K_{3} \times T^{2}, F_{h>1}$ vanishes and the prepotential is given by,

$$
\mathrm{F}\left(\mathrm{X}^{\mathrm{I}}, \mathrm{W}^{2}\right)=-\frac{1}{2} \mathrm{C}_{\mathrm{ab}} \frac{\mathrm{X}^{\mathrm{a}} \mathrm{X}^{\mathrm{b}} \mathrm{X}^{1}}{\mathrm{X}^{0}}-\frac{\mathrm{W}^{2}}{128 \pi \mathrm{i}} \log \Delta\left(\mathrm{e}^{2 \pi \mathrm{i} \frac{\mathrm{X}^{1}}{\mathrm{X}^{0}}}\right)
$$

where $\mathrm{C}_{\mathrm{ab}} \equiv \mathrm{C}_{1 \mathrm{ab}}$ with $\mathrm{a}, \mathrm{b}=2, \ldots, \mathrm{b}_{2}, \mathrm{~b}_{2}=23$, and $\mathrm{C}_{\mathrm{ABC}}$ being the intersection numbers of the $C Y_{3}$. $\Delta$ stands for the Dedekind function. Taking $\mathrm{p}^{0}=0$, the OSV free energy is given by,

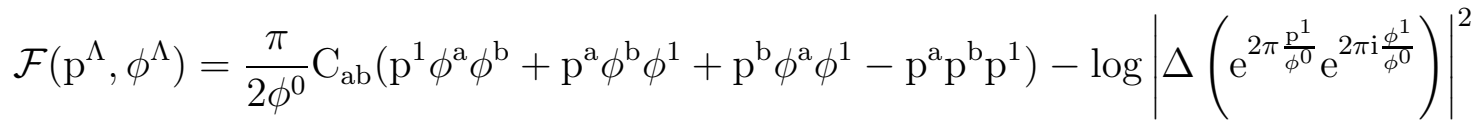

For the small black hole we set $p^{a}=0$,

$$
\mathcal{F}\left(\mathrm{p}^{\mathrm{I}}, \phi^{\mathrm{I}}\right)=\frac{1}{2} \mathrm{C}_{\mathrm{ab}} \frac{\phi^{\mathrm{a}} \phi^{\mathrm{b}} \mathrm{p}^{1}}{\phi^{0}}-\log \left(\left|\Delta\left(\mathrm{e}^{2 \pi^{2} \frac{\mathrm{p}^{1}}{\phi^{0}}} \mathrm{e}^{2 \pi \mathrm{i} \frac{\phi^{1}}{\phi^{0}}}\right)\right|^{2}\right)
$$

Accordingly the inverse Laplace transformation, with the Euclidean measure, calculates the degeneracy of states as,

$$
\begin{gathered}
\Omega\left(\mathrm{p}^{1}, \overrightarrow{\mathrm{q}}\right) \doteq \int\left[\mathrm{d} \phi^{0} \mathrm{~d} \phi^{1} \mathrm{~d} \phi^{\mathrm{a}}\right] \mathrm{e}^{\mathcal{F}+\mathrm{q}_{\mathrm{a}} \phi^{\mathrm{a}}+\mathrm{q}_{0} \phi^{0}+\mathrm{q}_{1} \phi^{1}} \\
\doteq \int \mathrm{d} \mathrm{xd} \theta\left(\frac{-\mathrm{x}^{12}\left(\mathrm{p}^{1}\right)^{2}}{\sqrt{\operatorname{det} \mathrm{C}}}\right) \frac{\mathrm{e}^{\left(\frac{1}{2} \mathrm{C}^{\mathrm{ab}} \mathrm{q}_{\mathrm{a}} \mathrm{qb}_{\mathrm{b}}-\mathrm{p}^{1} \mathrm{q}_{0}\right) \mathrm{x}-\mathrm{p}^{1} \mathrm{q}_{1} \mathrm{x} \theta}}{=}\left|\Delta\left(\mathrm{e}^{-\frac{2 \pi^{2}}{\mathrm{x}}} \mathrm{e}^{2 \pi \mathrm{i} \theta}\right)\right|
\end{gathered}
$$

\footnotetext{
†This example is in the context of $\mathcal{N}=4$, but for $\mathcal{N}=2$, where the OSV conjecture is originally formulated, this is the case as well. See for example the next section.
} 
where in the second line, we have taken the integration over $\phi^{a}$ 's and used the change of variables $x=-\phi^{0} / p^{1}$ and $\theta=\phi^{1} / \phi^{0}$ with $d \phi^{0} d \phi^{1}=\left(p^{1}\right)^{2} x d x d \theta$. For the two-charge case, we set $q_{A}=0$ for $A \neq 0$. Defining the T-duality invariant charge $N \equiv-p^{1} q_{0}$, the integrating over $\phi^{a}$ gives,

$$
\Omega\left(\mathrm{p}^{1}, \mathrm{q}_{0}\right) \doteq \frac{-\left(\mathrm{p}^{1}\right)^{2}}{\sqrt{\operatorname{det} \mathrm{C}}} \int \mathrm{dxx}{ }^{12} \mathrm{e}^{\mathrm{Nx}} \int \mathrm{d} \theta \frac{1}{\left|\Delta\left(\mathrm{e}^{-\frac{2 \pi^{2}}{\mathrm{x}}} \mathrm{e}^{2 \pi \mathrm{i} \theta}\right)\right|}
$$

In this form, beside the prefactor $\left(p^{1}\right)^{2}$, everything is in a duality invariant form. The presence of this $p$-dependent factor violates the duality invariance of the degeneracy of states.

In the large $N$ limit, $|\Delta(q)| \approx|q|$, so that $\Omega$ approaches,

$$
\Omega\left(\mathrm{p}^{1}, \mathrm{q}_{0}\right) \doteq \frac{-\left(\mathrm{p}^{1}\right)^{2}}{\sqrt{\operatorname{det} \mathrm{C}}} \hat{\mathrm{I}}_{13}(4 \pi \sqrt{\mathrm{N}})
$$

where [23],

$$
\hat{\mathrm{I}}_{\nu}(\mathrm{Q}) \equiv \frac{(2 \pi)^{\nu}}{\mathrm{i}} \int \mathrm{dt} \mathrm{t}^{-\nu-1} \mathrm{e}^{\mathrm{t}+\frac{\mathrm{Q}^{2}}{4 \mathrm{t}}}
$$

Note that at the above limit, the genus 0 and 1 terms of prepotential read as,

$$
\begin{gathered}
\mathrm{F}_{0}\left(\mathrm{X}^{\Lambda}\right)=-\frac{1}{2} \mathrm{C}_{\mathrm{ab}} \frac{\mathrm{X}^{1} \mathrm{X}^{\mathrm{a}} \mathrm{X}^{\mathrm{b}}}{\mathrm{X}^{0}} \\
\mathrm{~F}_{1}\left(\mathrm{X}^{\Lambda}\right)=-\frac{1}{64} \frac{\mathrm{X}^{1}}{\mathrm{X}^{0}}
\end{gathered}
$$

with the OSV free energy, being

$$
\mathcal{F}_{\mathrm{IIA} / \mathrm{K}_{3} \times \mathrm{T}^{2}}^{\mathrm{pert}}=\frac{\mathrm{C}_{\mathrm{ab}}}{2} \phi^{\mathrm{a}} \phi^{\mathrm{b}} \frac{\mathrm{p}^{1}}{\phi^{0}}-4 \pi^{2} \frac{\mathrm{p}^{1}}{\phi^{0}}
$$

We mention that any attempt to remove the prefactor of (2.9), and similar prefactors, should be done in a way to keep the correct results for the entropy and degeneracy of states. In the following sections we redefine the black hole ensemble to do this job.

\section{Redefinition of the OSV ensemble for a class of large and small black holes}

In this section we consider black holes with vanishing $D_{6}$-brane charge, where $\mathrm{p}^{0}=0$ and the prepotential takes a simplified form. We start with the case of large black holes . The perturbative OSV free energy for a general $C Y_{3}$ compactification in the large volume is given by,

$$
\mathcal{F}=-\frac{\pi}{6} \frac{\hat{\mathrm{C}}(\mathrm{p})}{\phi^{0}}+\frac{\pi}{2} \frac{\mathrm{C}_{\mathrm{AB}} \phi^{\mathrm{A}} \phi^{\mathrm{B}}}{\phi^{0}}
$$


where

$$
\mathrm{C}_{\mathrm{AB}}(\mathrm{p})=\mathrm{C}_{\mathrm{ABC}} \mathrm{p}^{\mathrm{C}}, \mathrm{C}(\mathrm{p})=\mathrm{C}_{\mathrm{ABC}} \mathrm{p}^{\mathrm{A}} \mathrm{p}^{\mathrm{B}} \mathrm{p}^{\mathrm{C}} ; \hat{\mathrm{C}}(\mathrm{p})=\mathrm{C}(\mathrm{p})+\mathrm{C}_{2 \mathrm{~A}} \mathrm{p}^{\mathrm{A}}
$$

and the indices $A, B, \ldots$ take the integers $1, \ldots, h$.

Let us change the variables of the free energy as follows,

$$
\psi^{0} \equiv \frac{6}{\hat{\mathrm{C}}(\mathrm{p})} \phi^{0} \quad ; \quad \frac{\mathrm{C}_{\mathrm{AB}}(\mathrm{p}) \phi^{\mathrm{A}} \phi^{\mathrm{B}}}{\phi^{0}} \equiv \frac{\psi^{\mathrm{A}} \psi^{\mathrm{A}}}{\psi^{0}}
$$

where $\psi^{\mathrm{A}}=\mathrm{M}_{\mathrm{B}}^{\mathrm{A}}(\mathrm{p}) \phi^{\mathrm{B}}$. $^{\mathrm{T}}$

The free energy is now redefined as a new function,

$$
\mathcal{F}=\grave{\mathcal{F}}\left(\psi^{0}, \psi^{\mathrm{A}}\right)=-\pi \frac{1}{\psi^{0}}+\frac{\pi}{2} \frac{\psi^{\mathrm{A}} \psi^{\mathrm{B}}}{\psi^{0}} .
$$

Noting that

$$
-\phi^{0} \frac{\partial \mathcal{F}}{\partial \phi^{0}}=-\psi^{0} \frac{\partial \grave{\mathcal{F}}}{\partial \psi^{0}} \quad ; \quad-\phi^{\mathrm{A}} \frac{\partial \mathcal{F}}{\partial \phi^{\mathrm{A}}}=-\psi^{\mathrm{A}} \frac{\partial \grave{\mathcal{F}}}{\partial \psi^{\mathrm{A}}}
$$

and defining

$$
\mathrm{N} \equiv \frac{1}{6} \hat{\mathrm{C}}(\mathrm{p}) \mathrm{q}_{0} \quad ; \quad \mathrm{N}_{\mathrm{A}} \equiv \mathrm{q}_{\mathrm{B}}\left(\mathrm{M}^{-1}\right)_{\mathrm{A}}^{\mathrm{B}}
$$

the Legendre transformation reads as,

$$
\begin{gathered}
\mathrm{S}\left(\mathrm{N}, \mathrm{N}_{\mathrm{A}}\right)=\grave{\mathcal{F}}-\pi \psi^{0} \frac{\partial \grave{\mathcal{F}}}{\partial \psi^{0}}-\pi \psi^{\mathrm{A}} \frac{\partial \grave{\mathcal{F}}}{\partial \psi^{\mathrm{A}}} \\
\mathrm{N}=-\frac{\partial \grave{\mathcal{F}}}{\partial \psi^{0}} \quad ; \quad \mathrm{N}_{\mathrm{A}}=-\frac{\partial \grave{\mathcal{F}}}{\partial \psi^{\mathrm{A}}} .
\end{gathered}
$$

Motivated by the above observation we introduce the correct black hole ensemble to use as

$$
\mathrm{Z} \equiv \mathrm{e}^{\grave{\mathcal{F}}\left(\psi^{0}, \psi^{\mathrm{A}}\right)}=\sum_{\mathrm{N}} \sum_{\mathrm{N}_{\mathrm{A}}} \tilde{\Omega}\left(\mathrm{N}, \mathrm{N}_{\mathrm{A}}\right) \mathrm{e}^{-\pi \psi^{0} \mathrm{~N}-\pi \psi^{\mathrm{A}} \mathrm{N}_{\mathrm{A}}}
$$

Note that the ensemble defined above, unlike the OSV ensemble, is pure canonical. Now the black hole degeneracy of states is proposed to be

$$
\mathrm{d}(\mathrm{p}, \mathrm{q}) \doteq \tilde{\Omega}\left(\mathrm{N}, \mathrm{N}_{\mathrm{A}}\right)
$$

Let us now consider (3.1) and calculate the corresponding degeneracy of states in the light of the definition (3.9),

$$
\tilde{\Omega}\left(\mathrm{N}, \mathrm{N}_{\mathrm{A}}\right) \doteq \int \mathrm{d} \psi^{0} \mathrm{~d} \psi^{\mathrm{A}} \mathrm{e}^{\grave{\mathcal{F}}\left(\psi^{0}, \psi^{\mathrm{A}}\right)+\pi \mathrm{N} \psi^{0}+\pi \mathrm{N}_{\mathrm{A}} \psi^{\mathrm{A}}}
$$

\footnotetext{
${ }^{\S} \mathrm{M}_{\mathrm{B}}^{\mathrm{A}}(\mathrm{p})$ is a transformation matrix which first diagonalizes $\mathrm{C}_{\mathrm{AB}}(\mathrm{p})$, then rescales the variables appropriately.
} 
The integral over the variables $\psi^{A}$ 's is Gaussian and yields

$$
\tilde{\Omega}\left(\mathrm{N}, \mathrm{N}_{\mathrm{A}}\right) \doteq \hat{\mathrm{N}}^{-\frac{\mathrm{h}}{2}-1} \int \mathrm{d} \mu \mu^{\frac{\mathrm{h}}{2}} \mathrm{e}^{\mu-\pi^{2} \frac{\hat{\mathrm{N}}}{\mu}}
$$

where

$$
\hat{\mathrm{N}} \equiv \mathrm{N}-\frac{1}{2} \mathrm{~N}_{\mathrm{A}} \mathrm{N}_{\mathrm{A}}
$$

A simple algebra shows that

$$
\hat{\mathrm{N}}=\frac{1}{6} \hat{\mathrm{C}}(\mathrm{p}) \hat{\mathrm{q}}_{0} \quad ; \quad \hat{\mathrm{q}}_{0} \equiv \mathrm{q}_{0}-\frac{1}{2} \mathrm{C}^{\mathrm{AB}} \mathrm{q}_{\mathrm{A}} \mathrm{q}_{\mathrm{B}}
$$

where $\mathrm{C}^{\mathrm{AB}}$ denotes the inverse of $\mathrm{C}_{\mathrm{AB}}$. Thus the degeneracy of the large black hole states as calculated via the ensemble (3.9) is given by,

$$
\mathrm{d}(\mathrm{p}, \mathrm{q}) \doteq \hat{\mathrm{I}}_{\frac{\mathrm{h}}{2}+1}(\mathrm{~S}) \quad ; \quad \mathrm{S}=2 \pi \sqrt{\frac{1}{6} \hat{\mathrm{C}}(\mathrm{p}) \hat{\mathrm{q}}_{0}}
$$

This is indeed the correct result. In contrast, the result by the standard OSV ensemble

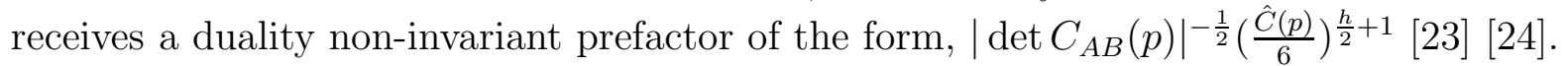

Now we come to the case of small black holes following the same idea: rewriting the OSV free energy and the macroscopic entropy in terms of new variables and new charges respectively leads to a new ensemble for the black holes which by construction is pure canonical. For the interesting case of $K 3$-fibrations with Heterotic duals, of which the most well-known example being $\frac{I I A}{K_{3} \times T^{2}} \equiv \frac{\text { Het. }}{T^{6}}$, the OSV free energy is (2.5). Defining

$$
\alpha \equiv-\frac{\phi^{0}}{\mathrm{p}^{1}} \quad ; \quad \beta \equiv \frac{\phi^{1}}{\mathrm{p}^{1}}
$$

and

$$
\mathrm{N} \equiv-\mathrm{p}^{1} \mathrm{q}_{0} \quad ; \quad \mathrm{N}^{\prime} \equiv-\mathrm{p}^{1} \mathrm{q}_{1}
$$

We obtain

$$
\grave{\mathcal{F}}\left(\alpha, \beta, \phi^{\mathrm{a}}\right)=-\frac{\mathrm{C}_{\mathrm{ab}} \phi^{\mathrm{a}} \phi^{\mathrm{b}}}{2 \alpha}-2 \log \left|\Delta\left(\mathrm{e}^{\frac{2 \pi^{2}}{\alpha}} \mathrm{e}^{\frac{-2 \pi \mathrm{i} \beta}{\alpha}}\right)\right|
$$

where

$$
\mathrm{S}\left(\mathrm{N}, \mathrm{N}^{\prime}, \mathrm{q}^{\mathrm{a}}\right)=\grave{\mathcal{F}}-\pi \alpha \frac{\partial \grave{\mathcal{F}}}{\partial \alpha}-\pi \beta \frac{\partial \grave{\mathcal{F}}}{\partial \beta}-\pi \phi^{\mathrm{a}} \frac{\partial \grave{\mathcal{F}}}{\partial \phi^{\mathrm{a}}}
$$

and

$$
\mathrm{N}=-\frac{\partial \grave{\mathcal{F}}}{\partial \alpha} \quad ; \quad \mathrm{N}^{\prime}=-\frac{\partial \grave{\mathcal{F}}}{\partial \beta} \quad ; \quad \mathrm{q}_{\mathrm{a}}=-\frac{\partial \grave{\mathcal{F}}}{\partial \phi^{\mathrm{a}}}
$$


Subsequently we define

$$
\grave{\mathrm{Z}} \equiv \mathrm{e}^{\grave{\mathcal{F}}\left(\alpha, \beta, \phi^{\mathrm{a}}\right)}=\sum_{\mathrm{N}} \sum_{\mathrm{N}^{\prime}} \sum_{\mathrm{q}_{\mathrm{a}}} \grave{\Omega}\left(\mathrm{N}, \mathrm{N}^{\prime}, \mathrm{q}_{\mathrm{a}}\right) \mathrm{e}^{-\pi \alpha \mathrm{N}-\pi \beta \mathrm{N}^{\prime}-\pi \phi^{\mathrm{a}} \mathrm{q}_{\mathrm{a}}}
$$

as the correct black hole ensemble.

The corresponding degeneracy of states is so given by,

$$
\grave{\Omega}\left(\mathrm{N}, \mathrm{N}^{\prime}, \mathrm{q}_{\mathrm{a}}\right) \doteq \int \mathrm{d} \alpha \mathrm{d} \beta \mathrm{d} \phi^{\mathrm{a}} \frac{\mathrm{e}^{-\frac{\mathrm{C}_{\mathrm{ab}} \phi^{\mathrm{a}} \phi^{\mathrm{b}}}{2 \alpha}+\pi \mathrm{N} \alpha+\pi \mathrm{N}^{\prime} \beta+\pi \mathrm{q}_{\mathrm{a}} \phi^{\mathrm{a}}}}{\left|\Delta\left(\mathrm{e}^{\frac{2 \pi 2}{\alpha}} \mathrm{e}^{\frac{-2 \pi \mathrm{i} \beta}{\alpha}}\right)\right|^{2}}
$$

Doing the Gaussian integration and changing the variables of the remaining integrals from $(\alpha, \beta)$ to $\left(\alpha, \theta \equiv-\frac{\beta}{\alpha}\right)$, we obtain

$$
\grave{\Omega}\left(\mathrm{N}, \mathrm{N}^{\prime}, \mathrm{q}_{\mathrm{a}}\right) \doteq \int \mathrm{d} \alpha \alpha^{12} \mathrm{e}^{\pi \alpha \hat{\mathrm{N}}} \int \mathrm{d} \theta \frac{\mathrm{e}^{-\pi \mathrm{N}^{\prime} \theta \alpha}}{\left|\Delta\left(\mathrm{e}^{\frac{2 \pi}{\alpha}} \mathrm{e}^{2 \pi \mathrm{i} \theta}\right)\right|^{2}}
$$

where

$$
\hat{\mathrm{N}} \equiv \mathrm{N}+\frac{1}{2} \mathrm{q}_{\mathrm{a}} \mathrm{C}^{\mathrm{ab}} \mathrm{q}_{\mathrm{b}}=\frac{1}{2} \mathrm{Q}_{\mathrm{e}}^{2}
$$

For the 2-charge case where $\hat{N}=N$ and $N^{\prime}=0$, taking the large-volume limit, for which $|\Delta(x)| \approx|x|$, simplifies (3.24) as

$$
\tilde{\Omega}(\mathrm{N}) \doteq \int \mathrm{d} \alpha \alpha^{12} \mathrm{e}^{\pi \alpha \hat{\mathrm{N}}+\frac{4 \pi}{\alpha}}=\hat{\mathrm{I}}_{13}(4 \pi \sqrt{\hat{\mathrm{N}}})
$$

which is the well known result for the degeneracy of states, as known from its duality with Heterotic string theory by direct counting of the DH fundamental stringy states [21].

\section{Black holes with non-vanishing $D_{6}$-brane charge: Saddle-point evaluation}

In this section we concern the most general case, $p^{0} \neq 0$, and as the central idea follow what we did before. We introduce a generalized OSV ensemble as,

$$
\begin{aligned}
& \grave{\mathrm{Z}} \equiv \mathrm{e}^{\grave{\mathcal{F}}\left(\mathrm{p}^{\Lambda}, \psi^{\Lambda}\right)}=\sum_{\grave{\mathrm{q}} \Lambda} \grave{\Omega}\left(\mathrm{p}^{\Lambda}, \grave{\mathrm{q}}_{\Lambda}\right) \mathrm{e}^{-\pi \grave{\mathrm{q}} \Lambda \psi^{\Lambda}} \\
& \grave{\mathcal{F}}\left(\mathrm{p}^{\Lambda}, \psi^{\Lambda}\right)=\mathcal{F}\left(\mathrm{p}^{\Lambda}, \phi^{\Lambda}\right)
\end{aligned}
$$

where the new variables $\psi^{\Lambda}$ which preserve the entropy-defining Legendre transformation,

$$
\mathrm{S}\left(\mathrm{p}^{\Lambda}, \grave{\mathrm{q}}_{\Lambda}\right)=\grave{\mathcal{F}}+\pi \grave{\mathrm{q}}_{\Lambda} \psi^{\lambda} \text {. }
$$


are defined from the electric potentials via the linear relations ",

$$
\psi^{\Lambda}=\mathrm{U}_{\Gamma}^{\Lambda}\left(\mathrm{p}^{\Sigma}, \phi_{*}^{\Sigma}\right) \phi^{\Gamma}=\mathrm{V}_{\Gamma}^{\Lambda}\left(\mathrm{p}^{\Sigma}, \mathrm{q}_{\Sigma}\right) \phi^{\Gamma}
$$

The fact that unlike the previous cases, here we let the matrix $U$ to depend on $\phi_{*}^{\Lambda}$ as well as $p^{\Lambda}$, roots in the observation that for the cases with non-vanishing $D_{6}$-brane charge, the OSV prefactors are functions of both the magnetic and electric charges [27][24].

We now require that the corresponding inverse Laplace integration as defined via the Euclidean measure,

$$
\grave{\Omega}\left(\mathrm{p}^{\Lambda}, \grave{\mathrm{q}}_{\Lambda}\right) \doteq \int\left[\mathrm{d} \psi^{\Lambda}\right] \mathrm{e}^{\grave{\mathcal{F}}+\pi \grave{\mathrm{q}}_{\Lambda} \psi^{\Lambda}}
$$

be duality invariant, exactly or at least at the saddle-point approximation. Subsequently the black hole degeneracy of states is recognized as,

$$
\mathrm{d}\left(\mathrm{p}^{\Lambda}, \mathrm{q}_{\Lambda}\right) \doteq \grave{\Omega}\left(\mathrm{p}^{\Lambda}, \grave{\mathrm{q}}_{\Lambda}\right)
$$

where as implied by (4.4),

$$
\grave{\mathrm{q}}_{\Lambda}=\mathrm{q}_{\Gamma}\left(\mathrm{V}^{-1}\right)^{\Gamma}{ }_{\Lambda}
$$

From (4.2) and (4.4), the relation between the ensemble (4.1) and (1.3) reads from ",

$$
\grave{\Omega} \doteq \int\left[\mathrm{d} \phi^{\Lambda}\right] \mathrm{J}(\psi \rightarrow \phi) \mathrm{e}^{\mathcal{F}+\pi \mathrm{q}_{\Lambda} \phi^{\Lambda}}=\operatorname{det}[\mathrm{V}] \int\left[\mathrm{d} \phi^{\Lambda}\right] \mathrm{e}^{\mathcal{F}+\pi \mathrm{q}_{\Lambda} \phi^{\Lambda}}=\operatorname{det}[\mathrm{V}] \Omega .
$$

Obviously a proper choice of the Jacobian matrix $V$ can remove any unwanted prefactor as obtained by the evaluation of (1.5). We so consider the cases where the result by (1.5) is duality-violating only by a (single) prefactor besides the proper result** Then for the duality invariance in the saddle-point asymptotic expansion of (4.8), a constraintequation on $U_{\Gamma}^{\Lambda}$ is formed as a necessary and sufficient condition. The exact form of this constraint may depends on the order of the saddle-point approximation. Here we write it for the first order. The saddle-point evaluation of (4.8) results at,

$$
\grave{\Omega}^{*}=\operatorname{det}(\mathrm{V}) \Omega^{*} .
$$

Thus at the first order,

$$
\begin{gathered}
\grave{\Omega}^{*} \doteq \operatorname{det}[\mathrm{V}] \operatorname{det}\left[\mathrm{H}_{*}\right]^{-\frac{1}{2}} \mathrm{e}^{\mathrm{S}} \\
\mathrm{H}_{\Lambda \Gamma} \equiv \frac{\partial^{2} \mathcal{F}}{\partial \phi^{\Lambda} \partial \phi^{\Gamma}} .
\end{gathered}
$$

\footnotetext{
IThe sign $*$ over or below a quantity stands for the saddle-point value of that quantity.

"Since the matrices $U$ and $V$ differ from each other just by a change of variable $q \leftrightarrow \phi_{*}$, hereafter both of them are denoted as $V$.

** Whether this is a general property of the OSV results, is a question which we address in the next section.
} 
Remembering that what we want to do is just to get rid of the unwanted prefactor appearing in $\Omega$ and to get a proper result, we define,

$$
\operatorname{det}\left[\mathrm{H}_{*}\right] \equiv \mathrm{S}^{\mathrm{m}} \operatorname{det}\left[\mathrm{H}_{*}^{\prime}\right]
$$

where $m$ denotes a convenient power of the entropy which is factorized out from the Hessian, so that $\operatorname{det}\left[\mathrm{H}_{*}^{\prime}\right]$ is the pure duality violating prefactor. To remove this prefactor by the matrix $\mathrm{V}$, we arrive at the constraint,

$$
\operatorname{det}\left[\mathrm{V}^{2}\right] \doteq \operatorname{det}\left[\mathrm{H}_{*}^{\prime}\right]
$$

The equation (4.13) admits infinite number of solutions, any solution of which is, in principle, as good as another ones. One choice is taking $V=v \mathbf{1}$ where ,

$$
\mathrm{v} \doteq\left(\operatorname{det}\left[\mathrm{H}_{*}^{\prime}\right]\right)^{\frac{1}{2 \mathrm{~h}+2}}
$$

As a different choice for $V$ we can take a diagonal matrix with elements $v_{\Lambda}$ such that,

$$
\mathrm{v}_{\Lambda} \doteq \sqrt{\mathrm{h}_{\Lambda}^{\prime}}
$$

where

$$
\prod_{\Lambda} \mathrm{h}_{\Lambda}^{\prime}=\operatorname{det}\left[\mathrm{H}_{*}^{\prime}\right]
$$

Equivalently, we can solve the the constraint (4.13) by defining the new ensemble to be

$$
\hat{\mathrm{X}}^{0} \equiv \operatorname{det}[\mathrm{V}] \mathrm{X}^{0} \quad ; \quad \hat{\mathrm{X}}^{\mathrm{A}} \equiv \mathrm{X}^{\mathrm{A}}
$$

This solution might be of particular physical interest since $\mathrm{X}^{0}$ controls the coupling of topological strings via the relation

$$
\mathrm{g}=\frac{4 \pi \mathrm{i}}{\mathrm{X}^{0}}
$$

In the conclusion we shall briefly speculate on a possible physical interpretation of the ensemble (4.17).

As a specific example in the sector $p^{0} \neq 0$, let us consider the case of a large black hole in type-IIA on a K3 fibered $C Y_{3}$, for which $C_{a b c}=C_{2 A}=0 ; a, b \in 2, \ldots, h$, as discussed in [24][27]. The prepotential is

$$
\mathcal{F}=\frac{1}{2}\left[\Im\left(\mathrm{t}^{1}\right) \mathrm{C}_{\mathrm{ab}}\left(\mathrm{p}^{\mathrm{a}} \mathrm{p}^{\mathrm{b}}-\phi^{\mathrm{a}} \phi^{\mathrm{b}}\right)+2 \Re\left(\mathrm{t}^{1}\right) \mathrm{p}^{\mathrm{a}} \mathrm{q}_{\mathrm{a}}\right]
$$

with $t^{A}=\frac{X^{A}}{X^{0}}$ being flat coordinates for the Kähler moduli. The Hessian-determinant of (4.19) at the attractor point is evaluated as,

$$
\operatorname{det}\left[\mathrm{H}_{*}\right] \doteq \mathrm{B}^{2}\left(\mathrm{C}_{\mathrm{ab}} \mathrm{p}^{\mathrm{a}} \mathrm{p}^{\mathrm{b}}-2 \mathrm{p}^{0} \mathrm{q}_{1}\right)^{\frac{\mathrm{h}}{2}-1} \mathrm{~S}^{-\frac{\mathrm{h}}{2}-2} \equiv \mathrm{S}^{-\frac{\mathrm{h}}{2}-2} \operatorname{det}\left[\mathrm{H}_{*}^{\prime}\right]
$$


where

$\mathrm{B}=\sqrt{\left(\mathrm{C}_{\mathrm{ab}} \mathrm{p}^{\mathrm{a}} \mathrm{p}^{\mathrm{b}}-2 \mathrm{p}^{0} \mathrm{q}_{1}\right)\left[\left(\mathrm{p}^{1}\right)^{2} \mathrm{C}_{\mathrm{ab}} \mathrm{p}^{\mathrm{a}} \mathrm{p}^{\mathrm{b}}+\left(\mathrm{p}^{0}\right)^{2} \mathrm{C}^{\mathrm{ab}} \mathrm{q}_{\mathrm{a}} \mathrm{q}_{\mathrm{b}}-2 \mathrm{p}^{0} \mathrm{p}^{1} \mathrm{p}^{\mathrm{a}} \mathrm{q}_{\mathrm{a}}\right]} ; \mathrm{C}^{\mathrm{an}} \mathrm{C}_{\mathrm{nb}}=\delta_{\mathrm{b}}^{\mathrm{a}}(4.21)$

Regarding (4.13), the new variables are defined via the constraint

$$
\operatorname{det}[\mathrm{V}] \doteq \mathrm{B}\left(\mathrm{C}_{\mathrm{ab}} \mathrm{p}^{\mathrm{a}} \mathrm{p}^{\mathrm{b}}-2 \mathrm{p}^{0} \mathrm{q}_{1}\right)^{\frac{\mathrm{h}-2}{4}}
$$

from which, in case of interest, one can exactly specify a new set of variables.

Finally we want to show how in a simple way the variables of (3.4) can be deduced from (4.15). The attractor point of the prepotential (3.1) is

$$
\left(\phi_{*}^{0}\right)^{2}=-\frac{1}{6} \frac{\hat{\mathrm{C}}(\mathrm{p})}{\hat{\mathrm{q}}_{0}} \quad ; \quad \phi_{*}^{\mathrm{A}}=-\mathrm{C}^{\mathrm{AB}}(\mathrm{p}) \mathrm{q}_{\mathrm{B}} \phi_{*}^{0}
$$

so that

$$
\operatorname{det}\left[\mathrm{H}_{*}\right] ; \doteq \operatorname{det}\left[\mathrm{C}_{\mathrm{AB}}(\mathrm{p})\right] \frac{\hat{\mathrm{C}}(\mathrm{p})}{\phi_{*}^{0}} \Rightarrow \operatorname{det}\left[\mathrm{H}_{*}^{\prime}\right] \doteq \operatorname{det}\left[\frac{\mathrm{C}_{\mathrm{AB}}(\mathrm{p})}{\hat{\mathrm{C}}(\mathrm{p})}\right] \hat{\mathrm{C}}^{-2}(\mathrm{p})
$$

and then from (4.15) we obtain

$$
\mathrm{v}^{0} \doteq \frac{1}{\hat{\mathrm{C}}(\mathrm{p})} \quad ; \quad \mathrm{v}^{\mathrm{A}} \doteq\left[\frac{\omega_{\mathrm{A}}(\mathrm{p})}{\hat{\mathrm{C}}(\mathrm{p})}\right]^{\frac{1}{2}}
$$

with $\omega_{A}(p)$ being the eigenvalues of $C_{A B}(p)$. Thus for the $\psi$ variables we obtain

$$
\psi^{0} \doteq \frac{\phi^{0}}{\hat{\mathrm{C}}(\mathrm{p})} \quad ; \quad \frac{\mathrm{C}_{\mathrm{AB}}(\mathrm{p}) \phi^{\mathrm{A}} \phi^{\mathrm{B}}}{\phi^{0}}=\frac{\omega_{\mathrm{A}}(\mathrm{p}) \hat{\phi}^{\mathrm{A}} \hat{\phi}^{\mathrm{A}}}{\phi^{0}} \doteq \frac{\psi^{\mathrm{A}} \psi^{\mathrm{A}}}{\psi^{0}}
$$

where $\hat{\phi}^{A}$ form a diagonal basis for $C_{A B}(p)$. The result (4.26) is in accordance with (3.3).

\section{$5 \quad$ Is a major modification needful?}

The electric-magnetic duality is restored by the ensemble (4.1), as long as the result by (1.5) admits the general form

$$
\Omega(\mathrm{p}, \mathrm{q}) \doteq \mathrm{N}(\mathrm{p}, \mathrm{q}) \mathrm{f}(\mathrm{S})
$$

We naturally ask if violation of the duality in the results of (1.5) is restricted only to a prefactor, $\mathrm{N}(\mathrm{p}, \mathrm{q})$. Of course for vanishing $\mathrm{D}_{6}$-brane charge, this is always true, at least for the large $C Y_{3}$-volume limit. In these cases the prepotential is quadratic with respect to $\phi^{\mathrm{A}}$, so the exact evaluation of (1.5) results at the form (5.1) with $\mathrm{f}(\mathrm{S})$ being a modified Bessel function. Now we come to the cases for which $\mathrm{p}^{0} \neq 0$. The prepotential, at the genus zero and one terms, is of the general form

$$
\mathcal{F}=\frac{\mathrm{p}^{0}}{\left(\phi^{0}\right)^{2}+\left(\mathrm{p}^{0}\right)^{2}}\left[\mathrm{E}(\phi)-3 \mathrm{E}_{\mathrm{AB}}(\phi) \mathrm{p}^{\mathrm{A}} \mathrm{p}^{\mathrm{B}}-\mathrm{E}_{2 \mathrm{~A}} \phi^{\mathrm{A}}\right]
$$




$$
+\frac{\phi^{0}}{\left(\phi^{0}\right)^{2}+\left(\mathrm{p}^{0}\right)^{2}}\left[\mathrm{E}(\mathrm{p})-3 \mathrm{E}_{\mathrm{AB}}(\mathrm{p}) \phi^{\mathrm{A}} \phi^{\mathrm{B}}+\mathrm{E}_{2 \mathrm{~A}} \mathrm{p}^{\mathrm{A}}\right]
$$

where

$$
\mathrm{E}(\mathrm{z}) \equiv \mathrm{E}_{\mathrm{ABC}} \mathrm{z}^{\mathrm{A}} \mathrm{Z}^{\mathrm{B}} \mathrm{Z}^{\mathrm{c}} ; \mathrm{E}_{\mathrm{AB}}(\mathrm{z}) \equiv \mathrm{E}_{\mathrm{ABC}} \mathrm{z}^{\mathrm{c}}
$$

with

$$
\mathrm{E}_{\mathrm{ABC}} \equiv-\frac{\pi}{6} \mathrm{C}_{\mathrm{ABC}} ; \mathrm{E}_{2 \mathrm{~A}} \equiv-\frac{\pi}{6} \mathrm{C}_{2 \mathrm{~A}}
$$

To address the mentioned question for the case of non-vanishing $\mathrm{p}^{0}$, we evaluate the OSV result for the black hole degeneracy of states in concrete examples, through incorporating all the terms which appear in the saddle-point asymptotic expansion of the integral (1.5),

$$
\begin{aligned}
\mathrm{G}=\mathrm{S}+\sum_{\mathrm{n}=2}^{\infty} \frac{1}{\mathrm{n} !} \mathrm{H}_{\Lambda_{1} \ldots \Lambda_{\mathrm{n}}}^{*} \eta^{\Lambda_{1}} \ldots \eta^{\Lambda_{\mathrm{n}}} \\
\mathrm{H}_{\Lambda_{1} \ldots \Lambda_{\mathrm{n}}} \equiv \frac{\partial^{\mathrm{n}} \mathcal{F}}{\partial \phi^{\Lambda_{1}} \ldots \partial \phi^{\Lambda_{\mathrm{n}}}} \\
\eta^{\Lambda} \equiv \phi^{\Lambda}-\phi_{*}^{\Lambda}
\end{aligned}
$$

for the two extreme limits: $\mathrm{p}^{0} \rightarrow \infty$ and $\mathrm{p}^{0} \rightarrow 0$.

\subsection{An example in the Large- $\mathrm{p}^{0}$ limit}

The prepotential (5.2) is expanded over $\frac{\phi^{0}}{\mathrm{p}^{0}}$ as

$$
\begin{aligned}
\mathcal{F}=\frac{1}{\mathrm{p}^{0}}\left\{\left[\mathrm{E}(\phi)-3 \mathrm{E}_{\mathrm{AB}}(\phi) \mathrm{p}^{\mathrm{A}} \mathrm{p}^{\mathrm{B}}-\mathrm{E}_{2 \mathrm{~A}} \phi^{\mathrm{A}}\right]\right. \\
\left.+\frac{\phi^{0}}{\mathrm{p}^{0}}\left[\mathrm{E}(\mathrm{p})-3 \mathrm{E}_{\mathrm{AB}}(\mathrm{p}) \phi^{\mathrm{A}} \phi^{\mathrm{B}}+\mathrm{E}_{2 \mathrm{~A}} \mathrm{p}^{\mathrm{A}}\right]+\mathrm{O}\left(\frac{\phi^{0}}{\mathrm{p}^{0}}\right)^{2}\right\} .
\end{aligned}
$$

We require that the contribution to (1.5) from the regions of integration far away from the attractor point is not major, and so ignoring terms of higher orders in the above expansion is satisfactory if $O\left(\left|\frac{\phi_{*}^{0}}{\mathrm{p}^{0}}\right|\right) \ll 1$. As we will see this would be the case for $\left|\frac{\mathrm{E}(\mathrm{p})}{\mathrm{p}^{0}}\right| \gg 1$.

For the limit under consideration, the prepotential is linear with respect to $\phi^{0}$, so (5.3) is easily evaluated as

$$
\begin{gathered}
\mathrm{G}(\eta)=\mathrm{S}-\frac{6}{\left(\mathrm{p}^{0}\right)^{2}} \mathrm{E}_{\mathrm{AB}}(\mathrm{p}) \mathrm{p}^{\mathrm{B}} \eta^{0} \eta^{\mathrm{A}} \\
+\frac{3}{\mathrm{p}^{0}}\left[\mathrm{E}_{\mathrm{AB}}\left(\phi_{*}\right)-\frac{\phi_{*}^{0}}{\mathrm{p}^{0}} \mathrm{E}_{\mathrm{AB}}(\mathrm{p})-\frac{\eta^{0}}{\mathrm{p}^{0}} \mathrm{E}_{\mathrm{AB}}(\mathrm{p})\right] \eta^{\mathrm{A}} \eta^{\mathrm{B}}+\frac{1}{\mathrm{p}^{0}} \mathrm{E}_{\mathrm{ABC}} \eta^{\mathrm{A}} \eta^{\mathrm{B}} \eta^{\mathrm{C}} \\
\equiv \mathrm{S}+\mathrm{g}_{\mathrm{A}} \eta^{\mathrm{A}}+\frac{1}{2} \mathrm{~g}_{\mathrm{AB}} \eta^{\mathrm{A}} \eta^{\mathrm{B}}+\mathrm{g}_{\mathrm{ABC}} \eta^{\mathrm{A}} \eta^{\mathrm{B}} \eta^{\mathrm{C}}
\end{gathered}
$$

Accordingly (1.5) leads to

$$
\Omega \doteq \mathrm{e}^{\mathrm{S}} \int \mathrm{d} \eta^{0} \int\left[\mathrm{d} \eta^{\mathrm{A}}\right] \mathrm{e}^{\mathrm{g}_{\mathrm{A}} \eta^{\mathrm{A}}+\frac{1}{2} \mathrm{~g}_{\mathrm{AB}} \eta^{\mathrm{A}} \eta^{\mathrm{B}}+\mathrm{g}_{\mathrm{ABC}} \eta \mathrm{A} \eta^{\mathrm{B}} \eta^{\mathrm{C}}}
$$




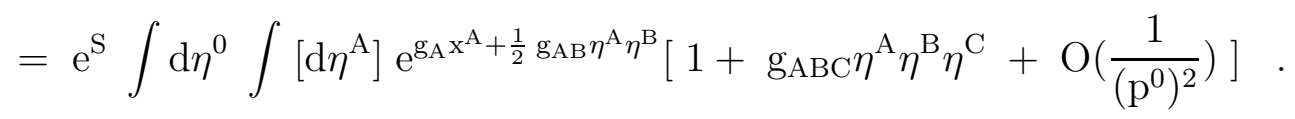

Now as a specific example of the case under consideration, we consider a charge configuration for which

$$
\phi_{*}^{\mathrm{A}}=\mathrm{p}^{\mathrm{A}} ; \phi_{*}^{0}=0
$$

which is in line with the attractor equations for

$$
\begin{gathered}
\mathrm{q}_{\mathrm{A}}=\frac{1}{\mathrm{p}^{0}} \mathrm{E}_{2 \mathrm{~A}} \\
\mathrm{q}_{0}=\frac{1}{\left(\mathrm{p}^{0}\right)^{2}}\left[2 \mathrm{E}(\mathrm{p})-\mathrm{E}_{2 \mathrm{~A}} \mathrm{p}^{\mathrm{A}}\right]
\end{gathered}
$$

with the entropy obtained as

$$
\mathrm{S}=-2 \frac{\mathrm{E}(\mathrm{p})}{\mathrm{p}^{0}}
$$

Given this ansatz, (1.5) is given by

$$
\begin{gathered}
\Omega \ddot{=} \mathrm{e}^{\mathrm{S}} \int \mathrm{d} \eta^{0}\left[\mathrm{~d} \eta^{\mathrm{A}}\right] \exp \left\{\frac{1}{2}\left[\frac{6}{\mathrm{p}^{0}}\left(1-\frac{\eta^{0}}{\mathrm{p}^{0}}\right) \mathrm{E}_{\mathrm{AB}}(\mathrm{p})\right] \eta^{\mathrm{A}} \eta^{\mathrm{B}}-\left[\frac{6}{\mathrm{p}^{0}} \frac{\eta^{0}}{\mathrm{p}^{0}} \mathrm{E}_{\mathrm{AB}}(\mathrm{p}) \mathrm{p}^{\mathrm{B}}\right] \eta^{\mathrm{A}}\right\}\left[1+\frac{1}{\mathrm{p}^{0}} \mathrm{E}(\eta)\right] \\
\equiv \Omega_{0}+\Omega_{1}
\end{gathered}
$$

where $\ddot{=}$ differs from $\doteq$ by higher order corrections. A simple algebra leads to the result

$$
\begin{aligned}
& \Omega_{1} \doteq \frac{\left(\mathrm{p}^{0}\right)^{\frac{\mathrm{h}}{2}+1}}{\sqrt{\operatorname{det}\left[\mathrm{E}_{\mathrm{AB}}\right]}} \mathrm{e}^{-2 \mathrm{~S}} \mathrm{I}_{\frac{\mathrm{h}}{2}-1}(3 \mathrm{~S}) \\
& \Omega_{2} \doteq \frac{\left(\mathrm{p}^{0}\right)^{\frac{\mathrm{h}}{2}+1}}{\sqrt{\operatorname{det}\left[\mathrm{E}_{\mathrm{AB}}\right]}} \mathrm{e}^{-2 \mathrm{~S}} \int \mathrm{dt} \mathrm{t}^{-\frac{\mathrm{h}}{2}}\left[\left(1+\frac{1}{\mathrm{t}}\right)^{3} \frac{\mathrm{S}}{2}-\frac{\mathrm{h}}{2} \frac{1-\mathrm{t}}{\mathrm{t}^{2}}\right] \mathrm{e}^{\frac{3}{2}\left(\mathrm{t}+\frac{1}{\mathrm{t}}\right) \mathrm{S}}
\end{aligned}
$$

so in this example $\Omega$ is of the form (5.1).

\subsection{The infinitesimal- $\mathrm{p}^{0}$ limit}

Here considering black holes with infinitesimal $\mathrm{D}_{6}$-brane charge, $\left|\mathrm{p}^{0}\right| \ll 1$, we evaluate the OSV degeneracy of states up to $O\left(\left|\mathrm{p}^{0}\right|^{2}\right)$. To do that, we do not restrict ourselves to any finite order of the saddle-point approximation and sum over all the terms of the series (5.3). However we still assume that the integral (1.5) is essentially localized around the saddle-point of the integrand, so that in the power expansion of the prepotential (5.2) over $\frac{\mathrm{p}^{0}}{\phi^{0}}$, we can ignore the terms $O\left(\frac{\mathrm{p}^{0}}{\phi^{0}}\right)^{2}$ as far as $\kappa \equiv \frac{\mathrm{p}^{0}}{\phi_{*}^{0}} \ll 1$. As the attractor-point equations will show us below, this assumption is consistent with the infinitesimal- $\mathrm{p}^{0}$ limit. The prepotential $(5.2)$, up to $O\left(\frac{\mathrm{p}^{0}}{\phi^{0}}\right)^{2}$, reads as

$$
\mathcal{F}=\frac{1}{\phi^{0}}\left\{\frac{\mathrm{p}^{0}}{\phi^{0}}\left[\mathrm{E}(\phi)-3 \mathrm{E}_{\mathrm{AB}}(\phi) \mathrm{p}^{\mathrm{A}} \mathrm{p}^{\mathrm{B}}-\mathrm{E}_{2 \mathrm{~A}} \phi^{\mathrm{A}}\right]\right.
$$




$$
\begin{aligned}
+[\mathrm{E}(\mathrm{p}) & \left.\left.-3 \mathrm{E}_{\mathrm{AB}}(\mathrm{p}) \phi^{\mathrm{A}} \phi^{\mathrm{B}}+\mathrm{E}_{2 \mathrm{~A}} \mathrm{p}^{\mathrm{A}}\right]\right\} \\
& \equiv \mathrm{p}^{0} \frac{\mathrm{K}}{\left(\phi^{0}\right)^{2}}+\frac{\mathrm{L}}{\phi^{0}} .
\end{aligned}
$$

Regarding that the prepotential is a polynomial of degree three with respect to $\phi^{\mathrm{A}}$ 's, we can explicitly evaluate the sum (5.3). Using

$$
\begin{aligned}
\mathrm{H}_{(\mathrm{n})}^{*} & =(-1)^{\mathrm{n}}\left[(\mathrm{n}+1) ! \mathrm{p}^{0} \frac{\mathrm{K}^{*}}{\left(\phi_{*}^{0}\right)^{\mathrm{n}+2}}+\mathrm{n} ! \frac{\mathrm{L}^{*}}{\left(\phi_{*}^{0}\right)^{\mathrm{n}+1}}\right] \\
\mathrm{H}_{\mathrm{A}(\mathrm{n})}^{*} & =(-1)^{\mathrm{n}}\left[(\mathrm{n}+1) ! \mathrm{p}^{0} \frac{\mathrm{K}_{\mathrm{A}}^{*}}{\left(\phi_{*}^{0}\right)^{\mathrm{n}+2}}+\mathrm{n} ! \frac{\mathrm{L}_{\mathrm{A}}^{*}}{\left(\phi_{*}^{0}\right)^{\mathrm{n}+1}}\right] \\
\mathrm{H}_{\mathrm{AB}(\mathrm{n})}^{*} & =(-1)^{\mathrm{n}}\left[(\mathrm{n}+1) ! \mathrm{p}^{0} \frac{\mathrm{K}_{\mathrm{AB}}^{*}}{\left(\phi_{*}^{0}\right)^{\mathrm{n}+2}}+\mathrm{n} ! \frac{\mathrm{L}_{\mathrm{AB}}^{*}}{\left(\phi_{*}^{0}\right)^{\mathrm{n}+1}}\right] \\
\mathrm{H}_{\mathrm{ABC}(\mathrm{n})}^{*} & =(-1)^{\mathrm{n}}\left[(\mathrm{n}+1) ! \mathrm{p}^{0} \frac{\mathrm{K}_{\mathrm{ABC}}^{*}}{\left(\phi_{*}^{0}\right)^{\mathrm{n}+2}}+\mathrm{n} ! \frac{\mathrm{L}_{\mathrm{ABC}}^{*}}{\left(\phi_{*}^{0}\right)^{\mathrm{n}+1}}\right]
\end{aligned}
$$

where

$$
\{K, L\}_{\left\{A_{1} \ldots A_{m}\right\}} \equiv \frac{\partial\{K, L\}}{\partial\left\{A_{1} \ldots A_{m}\right\}}
$$

and the index (n) denotes the number of derivatives with respect to $\phi^{0}$. Accordingly $\mathrm{G}(\eta)$ is evaluated as

$$
\begin{gathered}
\mathrm{G}(\eta)=\mathrm{S}+\left(\eta^{0}\right)^{2}\left[\sum_{0}^{\infty} \frac{\left(\eta^{0}\right)^{\mathrm{n}}}{(\mathrm{n}+2) !} \mathrm{H}_{(\mathrm{n}+2)}^{*}\right]+\eta^{0} \eta^{\mathrm{A}}\left[\sum_{0}^{\infty} \frac{\left(\eta^{0}\right)^{\mathrm{n}}}{(\mathrm{n}+1) !} \mathrm{H}_{\mathrm{A}(\mathrm{n}+1)}^{*}\right] \\
+\frac{1}{2} \eta^{\mathrm{A}} \eta^{\mathrm{B}}\left[\sum_{0}^{\infty} \frac{\left(\eta^{0}\right)^{\mathrm{n}}}{\mathrm{n} !} \mathrm{H}_{\mathrm{AB}(\mathrm{n})}^{*}\right]+\frac{1}{6} \eta^{\mathrm{A}} \eta^{\mathrm{B}} \eta^{\mathrm{C}}\left[\sum_{0}^{\infty} \frac{\left(\eta^{0}\right)^{\mathrm{n}}}{\mathrm{n} !} \mathrm{H}_{\mathrm{ABC}(\mathrm{n})}^{*}\right] \\
=\mathrm{S}+\frac{\left(\eta^{0}\right)^{2}}{\left(\phi_{*}^{0}\right)^{2}}\left[\frac{\mathrm{p}^{0} \mathrm{~K}^{*}}{\left(\phi_{*}^{0}\right)^{2}} \mathrm{R}_{3}\left(\frac{\eta^{0}}{\phi_{*}^{0}}\right)+\frac{\mathrm{L}^{*}}{\phi_{*}^{0}} \mathrm{R}\left(\frac{\eta^{0}}{\phi_{*}^{0}}\right)\right]-\frac{\eta^{0} \eta^{\mathrm{A}}}{\left(\phi_{*}^{0}\right)^{2}}\left[\frac{\mathrm{p}^{0} \mathrm{~K}_{\mathrm{A}}^{*}}{\phi_{*}^{0}} \mathrm{R}_{2}\left(\frac{\eta^{0}}{\phi_{*}^{0}}\right)+\mathrm{L}_{\mathrm{A}}^{*} \mathrm{R}\left(\frac{\eta^{0}}{\phi_{*}^{0}}\right)\right] \\
+\frac{1}{2} \frac{\eta^{\mathrm{A}} \eta^{\mathrm{B}}}{\left(\phi_{*}^{0}\right)^{2}}\left[\mathrm{p}^{0} \mathrm{~K}_{\mathrm{AB}}^{*} \mathrm{R}_{1}\left(\frac{\eta^{0}}{\phi_{*}^{0}}\right)+\phi_{*}^{0} \mathrm{~L}_{\mathrm{AB}}^{*} \mathrm{R}\left(\frac{\eta^{0}}{\phi_{*}^{0}}\right)\right]+\frac{1}{6} \frac{\eta^{\mathrm{A}} \eta^{\mathrm{B}} \eta^{\mathrm{C}}}{\left(\phi_{*}^{0}\right)^{3}} \mathrm{p}^{0} \phi_{*}^{0} \mathrm{~K}_{\mathrm{ABC}}^{*} \mathrm{R}_{1}\left(\frac{\eta^{0}}{\phi_{*}^{0}}\right)
\end{gathered}
$$

where

$$
\mathrm{R}(\mathrm{z}) \equiv \sum_{0}^{\infty}(-1)^{\mathrm{n}} \mathrm{z}^{\mathrm{n}}=\frac{1}{1+\mathrm{z}} ; \mathrm{R}_{\mathrm{m}}(\mathrm{z}) \equiv \sum_{0}^{\infty}(-1)^{\mathrm{n}}(\mathrm{n}+\mathrm{m}) \mathrm{z}^{\mathrm{n}}=\frac{(\mathrm{m}-1) \mathrm{z}+\mathrm{m}}{(1+\mathrm{z})^{2}} .
$$

Defining

$$
\mathrm{x} \equiv \frac{\eta^{0}}{\phi_{*}^{0}} ; \quad \mathrm{x}^{\mathrm{A}} \equiv \frac{\eta^{\mathrm{A}}}{\phi_{*}^{0}}
$$

together with

$$
\begin{gathered}
\mathrm{g}_{\mathrm{ABC}} \equiv \kappa \frac{\left(\phi_{*}^{0}\right)^{2}}{(1+\mathrm{x})^{2}} \mathrm{E}_{\mathrm{ABC}} \\
\mathrm{g}_{\mathrm{AB}} \equiv \frac{6 \phi_{*}^{0}}{(1+\mathrm{x})^{2}}\left[\kappa \mathrm{E}_{\mathrm{AB}}\left(\phi_{*}\right)-(1+\mathrm{x}) \mathrm{E}_{\mathrm{AB}}(\mathrm{p})\right]
\end{gathered}
$$




$$
\begin{aligned}
\mathrm{g}_{\mathrm{A}} \equiv-\frac{3 \mathrm{x}}{(1+\mathrm{x})^{2}}[\kappa(\mathrm{x} & \left.+2)\left\{\mathrm{E}_{\mathrm{AB}}\left(\phi_{*}\right) \phi_{*}^{\mathrm{B}}-\mathrm{E}_{\mathrm{AB}}(\mathrm{p}) \mathrm{p}^{\mathrm{B}}\right\}-2(1+\mathrm{x}) \mathrm{E}_{\mathrm{AB}}(\mathrm{p}) \phi_{*}^{\mathrm{B}}-\kappa \frac{\mathrm{x}+2}{3} \mathrm{E}_{2 \mathrm{~A}}\right] \\
\mathrm{g} \equiv & \frac{\mathrm{x}^{2}}{(1+\mathrm{x})^{2}} \frac{1}{\phi_{*}^{0}}\left[\left\{\mathrm{E}(\mathrm{p})(1+\mathrm{x})+\kappa \mathrm{E}\left(\phi_{*}\right)(3+2 \mathrm{x})\right\}\right. \\
& -3\left\{(1+\mathrm{x}) \mathrm{E}_{\mathrm{AB}}(\mathrm{p}) \phi_{*}^{\mathrm{A}} \phi_{*}^{\mathrm{B}}+\kappa(3+2 \mathrm{x}) \mathrm{E}_{\mathrm{AB}}\left(\phi_{*}\right) \mathrm{p}^{\mathrm{A}} \mathrm{p}^{\mathrm{B}}\right\} \\
+\quad & \left.+\left\{(1+\mathrm{x}) \mathrm{E}_{2 \mathrm{~A}} \mathrm{p}^{\mathrm{A}}-\kappa(3+2 \mathrm{x}) \mathrm{E}_{2 \mathrm{~A}} \phi_{*}^{\mathrm{A}}\right\}\right]
\end{aligned}
$$

we obtain

$$
\mathrm{G}(\eta)=\mathrm{S}+\mathrm{g}+\mathrm{g}_{\mathrm{A}} \mathrm{x}^{\mathrm{A}}+\frac{1}{2} \mathrm{~g}_{\mathrm{AB}} \mathrm{x}^{\mathrm{A}} \mathrm{x}^{\mathrm{B}}+\mathrm{g}_{\mathrm{ABC}} \mathrm{x}^{\mathrm{A}} \mathrm{x}^{\mathrm{B}} \mathrm{x}^{\mathrm{C}}
$$

Subsequently, (1.5) is given by

$$
\begin{aligned}
& \Omega \doteq \mathrm{e}^{\mathrm{S}}\left(\phi_{*}^{0}\right)^{\mathrm{h}+1} \int \mathrm{dx} \mathrm{e}^{\mathrm{g}} \int\left[\mathrm{dx}^{\mathrm{A}}\right] \mathrm{e}^{\mathrm{g}_{\mathrm{A}} \mathrm{x}^{\mathrm{A}}+\frac{1}{2} \mathrm{~g}_{\mathrm{AB}} \mathrm{x}^{\mathrm{A}} \mathrm{x}^{\mathrm{B}}+\mathrm{g}_{\mathrm{ABC}} \mathrm{x}^{\mathrm{A}} \mathrm{x}^{\mathrm{B}} \mathrm{x}^{\mathrm{C}}}
\end{aligned}
$$

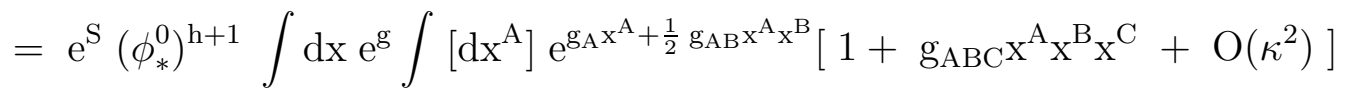

$$
\begin{aligned}
& \ddot{=} \mathrm{e}^{\mathrm{S}}\left(\phi_{*}^{0}\right)^{\mathrm{h}+1} \int \mathrm{dx} \frac{1}{\sqrt{\operatorname{det}\left[\mathrm{g}_{\mathrm{AB}}\right]}} \mathrm{e}^{\mathrm{g}}\left(1+\mathrm{g}_{\mathrm{ABC}} \frac{\partial^{3}}{\partial \mathrm{g}_{\mathrm{C}} \partial \mathrm{g}_{\mathrm{B}} \partial \mathrm{g}_{\mathrm{A}}}\right) \mathrm{e}^{-\frac{1}{2} \mathrm{~g}_{\mathrm{A}} \mathrm{g}^{\mathrm{AB}} \mathrm{g}_{\mathrm{B}}} \\
& \ddot{=} \mathrm{e}^{\mathrm{S}}\left(\phi_{*}^{0}\right)^{\mathrm{h}+1} \int \mathrm{dx} \frac{1}{\sqrt{\operatorname{det}\left[\mathrm{g}_{\mathrm{AB}}\right]}} \mathrm{e}^{\mathrm{T}_{1}}\left(1+\mathrm{T}_{2}+\mathrm{T}_{3}\right)
\end{aligned}
$$

where

$$
\begin{gathered}
\mathrm{T}_{1}=\mathrm{g}-\frac{1}{2} \mathrm{~g}_{\mathrm{A}} \mathrm{g}^{\mathrm{AB}} \mathrm{g}_{\mathrm{B}} \\
\mathrm{T}_{2}=3 \mathrm{~g}_{\mathrm{ABC}} \mathrm{g}^{\mathrm{AB}} \mathrm{g}^{\mathrm{CV}} \mathrm{g}_{\mathrm{V}} \\
\mathrm{T}_{3}=-\mathrm{g}_{\mathrm{ABC}} \mathrm{g}^{\mathrm{AM}} \mathrm{g}^{\mathrm{BN}} \mathrm{g}^{\mathrm{CV}} \mathrm{g}_{\mathrm{M}} \mathrm{g}_{\mathrm{N}} \mathrm{g}_{\mathrm{V}}
\end{gathered}
$$

and the sign '弚' meaning ' $\doteq$ ' up to $O\left(\kappa^{2}\right)$. Now to proceed further and check whether the above result is of the form (5.1), we need to know some concrete information about how $\mathrm{g}^{\mathrm{AB}}$ and $\mathrm{S}$ depend on $\mathrm{E}_{\mathrm{ABC}}, \mathrm{E}_{2 \mathrm{~A}}$ and the black hole charges. In that respect, a helpful choice for us is to set

$$
\phi_{*}^{\mathrm{A}}=\mathrm{p}^{\mathrm{A}}
$$

according to which, the attractor equations is equivalent with

$$
\begin{gathered}
\mathrm{q}_{\mathrm{A}}=\frac{6}{\phi_{*}^{0}} \mathrm{p}_{\mathrm{A}}+\frac{\mathrm{p}^{0}}{\left(\phi_{*}^{0}\right)^{2}} \mathrm{E}_{2 \mathrm{~A}} \\
\mathrm{q}_{0}=-\frac{1}{\left(\phi_{*}^{0}\right)^{2}}\left[2(1+2 \kappa) \mathrm{E}(\mathrm{p})-(1-2 \kappa) \mathrm{E}_{2 \mathrm{~A}} \mathrm{p}^{\mathrm{A}}\right]
\end{gathered}
$$

and the entropy reads as

$$
\mathrm{S}=\frac{2}{\phi_{*}^{0}}\left[(1-3 \kappa) \mathrm{E}(\mathrm{p})+(1-\kappa) \mathrm{E}_{2 \mathrm{~A}} \mathrm{p}^{\mathrm{A}}\right] .
$$


Now $\left\{\mathrm{g}, \mathrm{g}_{\mathrm{A}}, \mathrm{g}_{\mathrm{AB}}, \mathrm{T}_{1}, \mathrm{~T}_{2}, \mathrm{~T}_{3}\right\}$ is given by

$$
\begin{gathered}
\mathrm{g}_{\mathrm{AB}}=-6 \phi_{*}^{0} \frac{1+\mathrm{x}-\kappa}{(1+\mathrm{x})^{2}} \mathrm{E}_{\mathrm{AB}} \\
\mathrm{g}=\frac{\mathrm{x}^{2}}{(1+\mathrm{x})^{2}} \frac{1}{\phi_{*}^{0}}\left[-2 \mathrm{E}(\mathrm{p})\{1+\mathrm{x}+3 \kappa+2 \kappa \mathrm{x}\}+\mathrm{E}_{2 \mathrm{~A}} \mathrm{p}^{\mathrm{A}}\{1+\mathrm{x}-3 \kappa-2 \kappa \mathrm{x}\}\right] \\
\mathrm{T}_{1} \ddot{=} \frac{\mathrm{x}^{2}}{1+\mathrm{x}}\left[\left\{1-\kappa \frac{3+4 \mathrm{x}}{1+\mathrm{x}}\right\}+(1-\kappa) \mathrm{E}_{2 \mathrm{Ap}} \mathrm{A}\right] \frac{1}{\phi_{0}^{*}} \\
\mathrm{~T}_{2} \ddot{=} \kappa \frac{\mathrm{x}}{1+\mathrm{x}} \frac{\mathrm{h}}{2} \\
\mathrm{~T}_{3} \ddot{=} \kappa \frac{\mathrm{x}^{3}}{(1+\mathrm{x})^{2}} \frac{\mathrm{E}(\mathrm{p})}{\phi_{*}^{0}} .
\end{gathered}
$$

Thus the final expression for $\Omega$ is obtained as

$$
\begin{aligned}
\Omega & =\mathrm{e}^{\mathrm{S}}\left(\phi_{*}^{0}\right)^{\frac{\mathrm{h}}{2}+1} \frac{1}{\sqrt{\operatorname{det}\left[\mathrm{E}_{\mathrm{AB}}\right]}} \int \mathrm{dx}(1+\mathrm{x})^{\frac{\mathrm{h}}{2}} \mathrm{e}^{\mathrm{T}_{1}}\left[1+\kappa \frac{\mathrm{h}}{2(1+\mathrm{x})}+\mathrm{T}_{2}+\mathrm{T}_{3}\right] \\
& \ddot{=} \mathrm{e}^{\mathrm{S}}\left(\phi_{*}^{0}\right)^{\frac{\mathrm{h}}{2}+1} \frac{1}{\sqrt{\operatorname{det}\left[\mathrm{E}_{\mathrm{AB}}\right]}} \int \mathrm{dx}(1+\mathrm{x})^{\frac{\mathrm{h}}{2}} \mathrm{e}^{\mathrm{T}_{1}}\left[1+\kappa\left\{\frac{\mathrm{h}}{2}+\frac{\mathrm{x}^{2}}{(1+\mathrm{x})^{2}} \frac{\mathrm{E}(\mathrm{p})}{\phi_{0}^{*}}\right\}\right]
\end{aligned}
$$

Now from (5.5) and (5.6) it is obvious that (5.7) does not admit the form (5.1). Indeed if it was the case, it would be so for any h, implying that

$$
\int \mathrm{dx}(1+\mathrm{x})^{\frac{\mathrm{h}}{2}} \mathrm{e}^{\mathrm{T}_{1}}
$$

be itself of the form (5.1), which is not the case! ${ }^{\dagger}$

As we learn from the above examples, for black holes with non-vanishing $\mathrm{p}^{0}$ there is no guarantee that the result of (1.5) be of the form (5.1), if one incorporates the subleading terms of the saddle-point asymptotic expansion. As a consequence, even a generalization of type (1.6) or (4.8) does not restore the electric-magnetic duality. That is so because if the corresponding metric/Jacobian-determinant does not change the saddle-point of the integrand in (1.5), then it can not remove more than one non-invariant factor from the OSV result and if it changes the saddle-point of the integrand, then the leading term of the asymptotic expansion does not match microcanonically with the Bekenstein-HawkingWald entropy. It seems that this observation opens the possibility of deeper modifications.

\footnotetext{
${ }^{\dagger \dagger}$ The saddle-point is $\mathrm{x}=0$. At the leading order, the saddle approximation of (5.7) takes the form of (5.1), but this fails in the subleading orders.
} 


\section{$6 \quad$ An effective approach}

The ensemble defined through (4.1)-(4.4) is canonic in as many variables as the OSV ensemble, that is by construction the set of electric potentials is mapped one to one to the set of new variables $\psi^{\Lambda}$. However since the multiplet $\left(C X^{\Lambda}, C F_{\Lambda}\right)$ defines a vector under the symplectic transformations it is more natural for a black hole ensemble to treat both the magnetic and the electric charges at the same footing, if it is requested to produce symplectic invariant results. In that direction, the simplest generalization of (1.5) is an inverse Laplace transformation from: $Z_{\text {invari }} \equiv e^{\mathrm{G}}$, which integrates over both the $\Re X \equiv \mu$ and $\Im X \equiv \phi$ with an appropriate Jacobian/measure, such that

$$
\left[\mathrm{d} \mu_{\Lambda}\right]\left[\mathrm{d} \phi^{\Lambda}\right] \mathrm{J}(\mu, \phi)
$$

defines a symplectic invariant measure. In (6.1), $\mathrm{J}(\mu, \phi)$ appears either as a Jacobian when we change variables from those which originally define the ensemble to $(\mu, \phi)$ or as an intrinsic measure. As it is well known, one choice for the measure of (6.1) which is invariant under the symplectic transformations is,

$$
\left[\mathrm{d} \mu_{\Lambda}\right]\left[\mathrm{d} \phi^{\Lambda}\right] \operatorname{det}\left(\Im \mathrm{F}_{\Lambda \Gamma}\right)
$$

where

$$
\mathrm{F}_{\Lambda \Gamma} \equiv \frac{\partial^{2} \mathrm{~F}}{\partial \mathrm{X}^{\Lambda} \partial \mathrm{X}^{\Gamma}}
$$

(6.2) is used, for example, as the intrinsic measure of the ensemble introduced in [28]. However this measure vanishes for the case of $\frac{1}{2} \mathrm{BPS}$ black holes, so is not universally applicable. In fact a satisfactory universal measure has not been presented so far. Moreover even if we apply such a measure, to respect the electric-magnetic duality we need to introduce a symplectic invariant free energy $\mathrm{G}$. Thus regarding the fact that the OSV free energy, which as given by (1.3) and (1.4) forms the essence of the the OSV proposal, is only symplectic invariant at the attractor point, we take a more conservative approach in what follows. That is, to enjoy the proposed relationship between the topological-string free energy and the black hole physics within a manifest symmetric approach, we keep the OSV free energy unchanged but introduce an enlarged ensemble which is twice the OSV ensemble big, in the phase space terminology. This is done by associating to each doublet $\left(\mu^{\Lambda}, \phi^{\Lambda}\right)$, two canonical variables $\left(\chi_{\Lambda}, \zeta^{\Lambda}\right)$ which preserve the Legendre transformation from the OSV free energy to the black hole entropy. Obviously the simplest choice of such variables is to take them linear in $\phi^{\Lambda}$. Thus we define an invertible change of variables as,

$$
\chi_{\Lambda} \equiv \mathrm{A}(\mathrm{p}, \mathrm{q}) \phi^{\Lambda} \mathrm{f}_{\Lambda}\left(\mu^{\Lambda}\right) ; \quad \xi^{\Lambda} \equiv \mathrm{A}(\mathrm{p}, \mathrm{q}) \phi^{\Lambda} \mathrm{g}^{\Lambda}\left(\mu^{\Lambda}\right) ; \quad \forall \Lambda
$$

with

$$
\tilde{\mathcal{F}}\left(\chi_{\Lambda}, \xi^{\Lambda}\right)=\mathcal{F}\left(\mu^{\Lambda}, \phi^{\Lambda}\right)
$$

so that the Bekenstein-Hawking-Wald entropy is given by,

$$
\mathrm{S}\left(\mathrm{p}^{\Lambda}, \mathrm{q}_{\Lambda}\right)=\tilde{\mathrm{S}}\left(\alpha^{\Lambda}, \beta_{\Lambda}\right)=\tilde{\mathcal{F}}\left(\chi_{\Lambda}, \xi^{\Lambda}\right)+\pi \alpha^{\Lambda} \chi_{\Lambda}+\pi \beta_{\Lambda} \xi^{\Lambda}
$$




$$
-\alpha^{\Lambda}=\frac{\partial \tilde{\mathcal{F}}}{\partial \chi_{\Lambda}} \quad ; \quad-\beta_{\Lambda}=\frac{\partial \tilde{\mathcal{F}}}{\partial \xi^{\Lambda}}
$$

where the exact dictionary for translating the expressions in terms of the new charges $(\alpha, \beta)$ to those in terms of the black hole electric magnetic charges will be given a bit later in this section.

Based on (6.6) and (6.7), a black hole ensemble is defined as,

$$
\tilde{\mathrm{Z}} \equiv \mathrm{e}^{\tilde{\mathcal{F}}\left(\chi_{\Lambda}, \xi^{\Lambda}\right)}=\sum_{\alpha^{\Lambda}, \beta_{\Lambda}} \tilde{\Omega}\left(\alpha^{\Lambda}, \beta_{\Lambda}\right) \mathrm{e}^{-\pi \alpha^{\Lambda} \chi_{\Lambda}-\pi \beta_{\Lambda} \xi^{\Lambda}}
$$

As before we use the Euclidean measure for the ensemble (6.8), so that

$$
\mathrm{d}\left(\mathrm{p}^{\Lambda}, \mathrm{q}_{\Lambda}\right) \doteq \tilde{\Omega}\left(\alpha^{\Lambda}, \beta_{\Lambda}\right) \doteq \int\left[\mathrm{d} \chi_{\Lambda}\right]\left[\mathrm{d} \xi^{\Lambda}\right] \mathrm{e}^{\tilde{\mathcal{F}}+\pi \alpha^{\Lambda} \chi_{\Lambda}+\pi \beta_{\Lambda} \xi^{\Lambda}}
$$

Although the measure of (6.9),

$$
\prod_{\Lambda} \mathrm{d} \chi_{\Lambda} \mathrm{d} \xi^{\Lambda}=\mathrm{A}^{2}(\mathrm{p}, \mathrm{q}) \prod_{\Lambda} \phi^{\Lambda}\left(\mathrm{f}_{\Lambda} \mathrm{g}^{\prime \Lambda}-\mathrm{f}_{\Lambda}^{\prime} \mathrm{g}^{\Lambda}\right) \mathrm{d} \mu^{\Lambda} \mathrm{d} \phi^{\Lambda}
$$

is not universally symplectic invariant, unlike (6.2), we can follow the idea of the section 4: we require the asymptotic symplectic invariance of (6.9). More precisely, given an arbitrary order in the saddle-point asymptotic expansion of (6.9), we choose the function $\mathrm{A}(\mathrm{p}, \mathrm{q})$, in the definition (6.4), such that the unwanted prefactor of the corresponding OSV result is removed.

The integral (6.9) in terms of $(\mu, \phi)$ takes the form

$$
\mathrm{d}(\mathrm{p}, \mathrm{q}) \doteq \mathrm{A}^{2}(\mathrm{p}, \mathrm{q}) \int \prod_{\Lambda}\left[\mathrm{d} \phi^{\Lambda} \phi^{\Lambda}\right] \int \prod_{\Lambda}\left[\mathrm{d} \mu^{\Lambda}\left(\mathrm{f}_{\Lambda} \mathrm{g}^{\prime \Lambda}-\mathrm{f}_{\Lambda}^{\prime} \mathrm{g}^{\Lambda}\right)\right] \mathrm{Z}(\mu, \phi) \mathrm{e}^{\pi \mathrm{A} \phi^{\Lambda}\left(\alpha^{\Lambda} \mathrm{f}_{\Lambda}+\beta_{\Lambda} \mathrm{g}^{\Lambda}\right)}
$$

where $\mathrm{Z}(\mu, \phi)=\mathrm{e}^{\mathcal{F}(\mu, \phi)}$. Now to bridge between (6.11) and (1.5), we should effectively integrate over $\mu^{\Lambda}$ which fixes the value of $\mu^{\Lambda}$ at $\mu_{*}^{\Lambda}$. Here one important constraint on the functions $f_{\Lambda}$ and $g^{\Lambda}$ comes into play. First of all in accordance with (1.2) we require that the saddle-point values of $\mu^{\Lambda}$ 's coincide with the corresponding black hole magnetic charges,

$$
\mu_{*}^{\Lambda}=\mathrm{p}^{\Lambda} \quad ; \forall \Lambda
$$

Regarding the saddle-point equation for (6.11), (6.12) implies that

$$
\alpha^{\Lambda} \mathrm{f}_{\Lambda}^{\prime}(\mathrm{p})+\beta_{\Lambda} \mathrm{g}^{\prime \Lambda}(\mathrm{p})=0 ; \forall \Lambda
$$

Next we require that the saddle-point evaluation of (6.11) takes the form of

$$
\Omega\left(\mathrm{p}^{\Lambda}, \mathrm{q}^{\Lambda}\right) \doteq \int\left[\mathrm{d} \phi^{\Lambda}\right] \mathrm{M}(\mathrm{p}, \mathrm{q}, \phi) \mathrm{e}^{\mathcal{F}+\pi \mathrm{q}_{\Lambda} \phi^{\Lambda}}
$$

which together with (6.12) imply that

$$
\frac{\mathrm{q}_{\Lambda}}{\mathrm{A}(\mathrm{p}, \mathrm{q})}=\alpha^{\Lambda} \mathrm{f}_{\Lambda}(\mathrm{p})+\beta_{\Lambda} \mathrm{g}^{\Lambda}(\mathrm{p}) \quad ; \forall \Lambda \quad
$$


The dictionary between $(\alpha, \beta)$ and $(\mathrm{p}, \mathrm{q})$ is now given by the solution to the equations (6.13) and (6.15), which reads as

$$
\begin{aligned}
& \alpha^{\Lambda}=\frac{q_{\Lambda}}{A(p, q)} \frac{g^{\prime \Lambda}(p)}{f_{\Lambda}(p) g^{\prime \Lambda}(p)-f_{\Lambda}^{\prime}(p) g^{\Lambda}(p)} \\
& \beta_{\Lambda}=\frac{q_{\Lambda}}{A(p, q)} \frac{-f^{\prime \Lambda}(p)}{f_{\Lambda}(p) g^{\prime \Lambda}(p)-f_{\Lambda}^{\prime}(p) g^{\Lambda}(p)}
\end{aligned}
$$

Now as a constraint on the functions $\mathrm{f}$ and $\mathrm{g}$, we require that the equations (6.16) and (6.17) are consistent with the attractor equations of (6.7), for $\mathrm{A}(\mathrm{p}, \mathrm{q})$ treated as an arbitrary given function. That is, the following two equations should hold

$$
\begin{aligned}
\frac{\partial \tilde{\mathcal{F}}}{\partial \chi_{\Lambda}^{*}} & =-\frac{\mathrm{q}_{\Lambda}}{\mathrm{A}(\mathrm{p}, \mathrm{q})} \frac{\mathrm{g}^{\prime \Lambda}(\mathrm{p})}{\mathrm{f}_{\Lambda}(\mathrm{p}) \mathrm{g}^{\prime \Lambda}(\mathrm{p})-\mathrm{f}_{\Lambda}^{\prime}(\mathrm{p}) \mathrm{g}^{\Lambda}(\mathrm{p})} \\
\frac{\partial \tilde{\mathcal{F}}}{\partial \xi_{*}^{\Lambda}} & =\frac{\mathrm{q}_{\Lambda}}{\mathrm{A}(\mathrm{p}, \mathrm{q})} \frac{\mathrm{f}^{\prime \Lambda}(\mathrm{p})}{\mathrm{f}_{\Lambda}(\mathrm{p}) \mathrm{g}^{\prime \Lambda}(\mathrm{p})-\mathrm{f}_{\Lambda}^{\prime}(\mathrm{p}) \mathrm{g}^{\Lambda}(\mathrm{p})}
\end{aligned}
$$

with $\chi_{\Lambda}^{*}=\mathrm{A} \phi_{*}^{\Lambda} \mathrm{f}_{\Lambda}\left(\mathrm{p}^{\Lambda}\right), \xi_{*}^{\Lambda}=\mathrm{A} \phi_{*}^{\Lambda} \mathrm{g}^{\Lambda}\left(\mathrm{p}^{\Lambda}\right)$ and $\mathrm{q}_{\Lambda}=-\frac{1}{\pi} \frac{\partial \mathcal{F}}{\partial \phi_{*}^{\Lambda}}$.

Given these requirements, the first order saddle-point evaluation of (6.11) takes the form of (6.14) with

$$
\mathrm{M}(\mathrm{p}, \mathrm{q}, \phi)=\mathrm{A}^{2 \mathrm{~h}+2}(\mathrm{p}, \mathrm{q}) \prod_{\Lambda}\left[\left(\frac{\phi^{\Lambda}}{\mathrm{q}_{\Lambda}}\right)^{\frac{1}{2}} \frac{\left(\mathrm{f}_{\Lambda}(\mathrm{p}) \mathrm{g}^{\prime \Lambda}(\mathrm{p})-\mathrm{g}^{\Lambda}(\mathrm{p}) \mathrm{f}_{\Lambda}^{\prime}(\mathrm{p})\right)^{\frac{3}{2}}}{\left(\mathrm{f}_{\Lambda}^{\prime \prime}(\mathrm{p}) \mathrm{g}^{\prime \Lambda}(\mathrm{p})-\mathrm{g}^{\prime \prime} \Lambda(\mathrm{p}) \mathrm{f}_{\Lambda}^{\prime}(\mathrm{p})\right)^{\frac{1}{2}}}\right] .
$$

So, the effective inverse Laplace transformation, (6.14), differs from the original OSV formula, (1.5), by a measure $M$ which in form is something between the metric measure of (1.6) and the Jacobian-matrix in (4.8).

Now given a specific prepotential and an arbitrary order of the saddle-point asymptotic expansion of (6.14), the measure (6.20) equals

$$
\begin{gathered}
\grave{M}(\mathrm{p}, \mathrm{q}) \equiv \mathrm{M}\left(\mathrm{p}, \mathrm{q}, \phi_{*}\right)=\mathrm{A}^{2 \mathrm{~h}+2}(\mathrm{p}, \mathrm{q}) \prod_{\Lambda}\left[\frac{\left(\mathrm{f}_{\Lambda}(\mathrm{p}) \mathrm{g}^{\prime \Lambda}(\mathrm{p})-\mathrm{g}^{\Lambda}(\mathrm{p}) \mathrm{f}_{\Lambda}^{\prime}(\mathrm{p})\right)^{\frac{3}{2}}}{\left(\mathrm{f}_{\Lambda}^{\prime \prime}(\mathrm{p}) \mathrm{g}^{\prime \Lambda}(\mathrm{p})-\mathrm{g}^{\prime \prime}(\mathrm{p}) \mathrm{f}_{\Lambda}^{\prime}(\mathrm{p})\right)^{\frac{1}{2}}} \sqrt{\frac{\phi_{*}^{\Lambda}}{\mathrm{q}_{\Lambda}}}\right] \\
\tilde{\Omega}_{*}=\grave{\mathrm{M}}(\mathrm{p}, \mathrm{q}) \Omega_{*} .
\end{gathered}
$$

It is obvious that $\grave{M}(p, q)$ plays the same role as $\operatorname{det}[V]$ of the section 4 and protects the resulting black hole degeneracy of states against the electric-magnetic duality violations by removing the unwanted prefactors, with a proper choice of the function $A(p, q)$. 


\section{Conclusion}

We studied the issue of dualities in the OSV proposal. We showed that, as far as the duality-violation of the OSV result for the degeneracy of states is restricted to a prefactor, through a redefinition of the ensemble in terms of proper variables, one obtains the desired duality properties. In these cases, the degeneracy of states which comes from the inverse Laplace transformation with a flat measure of integration, is free from any unwanted prefactor which appears in ordinary OSV mixed ensemble. The result shows harmony with the microscopic results if we are careful about the validity of the perturbative regime and the application domain of the conjecture, at least to the extent that we know about the microscopic results. For the case of vanishing $D_{6}$-brane charge, where the ensemble is pure canonical and the restoration of the duality is exact, our procedure shows that the measure in the phase space of the black hole is intrinsically flat. However this is not always the case for the case $\mathrm{p}^{0} \neq 0$. Finally from our observations the following questions call for addressing.

1. In all these proposals, we have no natural choice for these variables, however, we can choose them phenomenologically. Understanding and interpreting these variables need further investigations.

2. To define the ensemble of sections 3 and 4, one can absorb the unwanted OSV prefactor within the redefinition of a single $\mathrm{X}$ variable. Among the $\mathrm{X}$ variables, $\mathrm{X}^{0}$ which controls the topological-string coupling via (4.18), plays a distinguished role in the prepotential (5.2). It is physically natural to think about a redefinition of the OSV ensemble as $\hat{\mathrm{X}}^{0}=\grave{\mathrm{M}}(\mathrm{p}, \mathrm{q}) \mathrm{X}^{0}$, according to (4.17), and ask if for a given black hole charge multiplet $\left(\mathrm{p}^{\Lambda}, \mathrm{q}_{\Lambda}\right)$, the requirement of the electric-magnetic duality gives an effective sense to the topological-string coupling as seen by the black hole ensemble through the identification $\hat{\mathrm{g}} \equiv \frac{4 \pi \mathrm{i}}{\hat{\mathrm{X}}^{0}}$.

3. Considering black holes with non-vanishing $D_{6}$-brane charge, there are cases where no measure refinement as (1.6) can restore the electric-magnetic duality if one incorporates the subleading terms of the asymptotic expansion. Do we need to go for a deeper modification of the OSV proposal?

\section{Acknowledgments}

We wish to thank Ashoke Sen for motivating us to address this problem and for valuable discussions. We would like to thank Cumrun Vafa and Atish Dabholkar for useful comments. A. T. thanks Hirosi Ooguri for nice discussions during the ICTP Spring School on Superstring Theory and Related Topics, 2005. 


\section{References}

[1] E. Witten, "Topological Sigma Models," Commun. Math. Phys. 118, 411 (1988).

[2] E. Witten, "On The Structure Of The Topological Phase Of Two-Dimensional Gravity," Nucl. Phys. B 340, 281 (1990).

[3] E. Witten, "Mirror manifolds and topological field theory," arXiv:hep-th/9112056.

[4] K. Hori et al. (Editors), "Mirror Symmetry" ( Clay Mathematics Monographs, v. 1), ISSN: 1539-6061, 2003.

[5] A. Neitzke and C. Vafa, "Topological strings and their physical applications," arXiv:hep-th/0410178.

[6] M. Vonk, "A mini-course on topological strings," arXiv:hep-th/0504147.

[7] M. Marino, "Les Houches lectures on matrix models and topological strings," arXiv:hep-th/0410165.

[8] A. Strominger and C. Vafa, "Microscopic Origin of the Bekenstein-Hawking Entropy," Phys. Lett. B 379, 99 (1996) [arXiv:hep-th/9601029].

[9] H. Ooguri, A. Strominger and C. Vafa, "Black hole attractors and the topological string," Phys. Rev. D 70, 106007 (2004) [arXiv:hep-th/0405146].

[10] R. M. Wald, "Black hole entropy in the Noether charge," Phys. Rev. D 48, 3427 (1993) [arXiv:gr-qc/9307038].

[11] G. Lopes Cardoso, B. de Wit and T. Mohaupt, "Corrections to macroscopic supersymmetric black-hole entropy," Phys. Lett. B 451, 309 (1999) [arXiv:hep-th/9812082].

[12] G. Lopes Cardoso, B. de Wit and T. Mohaupt, "Deviations from the area law for supersymmetric black holes," Fortsch. Phys. 48, 49 (2000) [arXiv:hep-th/9904005].

[13] J. M. Maldacena, A. Strominger and E. Witten, 'Black hole entropy in M-theory," JHEP 9712, 002 (1997) [arXiv:hep-th/9711053].

[14] T. Mohaupt, "Black hole entropy, special geometry and strings," Fortsch. Phys. 49, 3 (2001) [arXiv:hep-th/0007195].

[15] M. Bershadsky, S. Cecotti, H. Ooguri and C. Vafa, "Kodaira-Spencer theory of gravity and exact results for quantum string Commun. Math. Phys. 165, 311 (1994) [arXiv:hep-th/9309140].

[16] I. Antoniadis, E. Gava, K. S. Narain and T. R. Taylor, "Topological amplitudes in string theory," Nucl. Phys. B 413, 162 (1994) [arXiv:hep-th/9307158].

[17] R. Dijkgraaf, R. Gopakumar, H. Ooguri and C. Vafa, "Baby universes in string theory," arXiv:hep-th/0504221. 
[18] M. Aganagic, H. Ooguri, N. Saulina and C. Vafa, "Black holes, q-deformed 2d Yang-Mills, and non-perturbative topological strings," Nucl. Phys. B 715, 304 (2005) [arXiv:hep-th/0411280].

$[19]$

[19] E. P. Verlinde, "Attractors and the holomorphic anomaly," arXiv:hep-th/0412139.

[20] D. Shih and X. Yin, "Exact Black Hole Degeneracies and the Topological String," arXiv:hep-th/0508174.

[21] A. Dabholkar, "Exact counting of black hole microstates," arXiv:hep-th/0409148.

[22] H. Ooguri, "Topological String Theory," , STRINGS05, TORONTO, July 11, 2005, [http://www.fields.utoronto.ca/audio/05-06/strings/ooguri/].

[23] A. Dabholkar, F. Denef, G. W. Moore and B. Pioline, "Exact and asymptotic degeneracies of small black holes," arXiv:hep-th/0502157.

[24] A. Dabholkar, F. Denef, G. W. Moore and B. Pioline, "Precision counting of small black holes," arXiv:hep-th/0507014.

[25] R. Dijkgraaf, S. Gukov, A. Neitzke and C. Vafa, "Topological M-theory as unification of form theories of gravity," arXiv:hep-th/0411073.

[26] V. Pestun and E. Witten, "The Hitchin functionals and the topological B-model at one loop," arXiv:hep-th/0503083.

[27] B. Pioline, "BPS black hole degeneracies and minimal automorphic representations," arXiv:hep-th/0506228.

[28] B. de Wit, "Supersymmetric Black Hole Partition Functions," STRINGS05, TORONTO, July 11, 2005, [http://www.fields.utoronto.ca/audio/05-06/strings/wit/] .

[29] A. Sen, "How does a fundamental string stretch its horizon?," JHEP 0505, 059 (2005) [arXiv:hep-th/0411255].

[30] A. Sen, "Black holes, elementary strings and holomorphic anomaly," arXiv:hepth/0502126.

[31] A. Sen, "Black holes and the spectrum of half-BPS states in $\mathrm{N}=4$ supersymmetric string theory," arXiv:hep-th/0504005. 